Homeomorfismos do toro cujo conjunto de rotação é um segmento de reta

Romenique da Rocha Silva 



\title{
Homeomorfismos do toro cujo conjunto de rotação é um segmento de reta
}

\author{
Romenique da Rocha Silva
}

Orientador: Carlos Alberto Maquera Apaza

Dissertação apresentada ao Instituto de Ciências Matemáticas e de Computação - ICMC-USP, como parte dos requisitos para obtenção do título de Mestre em Ciências - Matemática.

\author{
USP - São Carlos \\ Julho/2007
}



Aos meus pais,

Ranulfo Florencio da Silva e Margarida Maria da Rocha, e minhas "meninas", Lília Belém de Assunção e Letícia Assunção Silva 



\section{Agradecimentos}

A José Carlos e Joatan Santos, pessoas que foram fundamentais para que eu iniciasse o mestrado.

Ao professor Carlos Maquera, pela orientação.

A todos os meus colegas e amigos do ICMC-USP, que tornaram os dias mais agradáveis.

A minha família, que esteve comigo nos momentos mais difíceis, Lília e Letícia.

Ao $\mathrm{CNPq}$, pelo apoio financeiro recebido durante o mestrado. 



\section{Resumo}

Um dos teoremas conhecidos de Poincaré afirma: Seja $f$ um homeomorfismo do círculo que preserva orientação. Se $p / q, \operatorname{com} \operatorname{mdc}(p, q)=1$, é o número de rotação de $f$, então f possui um ponto periódico de período q. Quando o conceito de número de rotação para um homeomorfismo do círculo é generalizado para um homeomorfismo $f: \mathbb{T}^{2} \rightarrow \mathbb{T}^{2}$ homotópico à identidade, o resultado é um subconjunto convexo do plano $\mathbb{R}^{2}$, chamado conjunto de rotação e é denotado por $\rho(F)$ onde $F$ é um levantamento de $f$. No caso que $\rho(F)$ tem interior não vazio, J. Franks obteve resultados análogos ao Teorema de Poincaré. Nesta dissertação estudamos um resultado análogo, obtido por Jonker e Zhang, quando $\rho(F)$ não tem interior. Mais precisamente: assumimos que $\rho(F)$ é um segmento de reta

com inclinação irracional e mostramos que se $\frac{1}{n}\left(p_{1}, p_{2}\right) \in \rho(F), \operatorname{com} \operatorname{mdc}\left(p_{1}, p_{2}, n\right)=1$, então $f$ possui um ponto periódico de período $n$. 



\section{Abstract}

One of the well know results of Poincaré state: Let $f$ be an orientation preserving circle homeomorphism. If $p / q$, with $\operatorname{mdc}(p, q)=1$, is the rotation number of $f$, then there is a periodic point for $f$ whose period is $q$. When the concept of rotations number, for orientation preserving circle homeomorphism, is generalized for torus homeomorphism $f: \mathbb{T}^{2} \rightarrow \mathbb{T}^{2}$ that are homotopic to the identity, it results in a convex subset of $\mathbb{R}^{2}$, called rotation set and is denoted by $\rho(F)$ where $F$ is a lifting of $f$. In the case that $\rho(F)$ has non-empty interior, J. Franks proved similar results to the Poincaré Theorem. In this work, when $\rho(F)$ has empty interior, we study an similar result obtained by Jonker and Zhang. More precisely: they suppose that the rotation set $\rho(F)$ is a line segment with irrational slope and demonstrate that if $\frac{1}{n}\left(p_{1}, p_{2}\right) \in \rho(F)$, with $\operatorname{mdc}\left(p_{1}, p_{2}, n\right)=1$, then $f$ has a periodic point of period $n$. 



\section{Lista de Figuras}

1.1 Grafo. . . . . . . . . . . . . . . . . . . 6

1.2 Grafo orientado. . . . . . . . . . . . . . . . 6

2.1 Imagem da reta $r$ pela aplicação $\pi \ldots \ldots$. . . . . . . . . . . 22

2.2 Anel essencial no toro $\mathbb{T}^{2} \ldots \ldots \ldots \ldots \ldots \ldots$

$2.3 \pi^{-1}\left(M_{j}\right)$ e as retas paralelas com a mesma inclinação de $\widetilde{M}_{j} \ldots \ldots \ldots$. . . 25

3.1 E-partição de $I^{2} \ldots \ldots \ldots \ldots \ldots \ldots$

3.2 Caminho orientado $\gamma=\left(e_{i_{0} i_{1}}, e_{i_{1} i_{2}}, \ldots, e_{i_{k-1} i_{k}}\right)$ em $G_{n}(\varepsilon) \ldots \ldots$

3.3 Segmento com inclinação racional. . . . . . . . . . . . . . . . . . . 38

3.4 O conjunto $E(v, \theta, r) \ldots \ldots \ldots \ldots \ldots \ldots \ldots \ldots$

3.5 O conjunto $E(\omega, \theta, r) \cup E(v, \theta, r) \ldots \ldots \ldots \ldots \ldots$

3.6 Campo de vetores $\tilde{X}$. . . . . . . . . . . . . . . . . . . 43 



\section{Lista de Símbolos}

$$
\begin{array}{ll}
\mathbb{Z} & \text { Conjunto dos números inteiros } \\
\mathbb{Q} & \text { Conjunto dos números racionais } \\
\mathbb{Q}_{+}=\{x \in \mathbb{Q} ; x \geq 0\} & \mathbb{Z}_{+}=\{0,1,2, \ldots\} \\
\mathbb{N}=\{1,2,3, \ldots\}
\end{array}
$$





\section{Sumário}

$\begin{array}{ll}\text { Introdução } & 1\end{array}$

1 Preliminares 5

1.1 Conceitos e resultados importantes . . . . . . . . . . . . . . 5

1.2 Homeomorfismos do círculo e número de rotação . . . . . . . . . . . . . . . 6

2 Conjunto de rotação e transitividade por cadeias $\quad 15$

2.1 Conjunto de rotação: propriedades gerais . . . . . . . . . . . . . . 15

2.2 Recorrência e transitividade por cadeias . . . . . . . . . . . . . . 18

3 Conjunto de rotação e pontos periódicos $\quad 27$

3.1 O grafo orientado $G_{n}(\varepsilon)$ de uma $\varepsilon$-partição . . . . . . . . . . . . . 28

3.1.1 Caminhos e laços orientados em $G_{n}(\varepsilon) \ldots \ldots$. . . . . . . . 30

3.1.2 A componente de 0 em $\mathbb{Z}^{2}$ por $\varepsilon$-cadeias . . . . . . . . . . . 34

3.2 Prova do Teorema Principal . . . . . . . . . . . . . . . . . . . . . 40

Referências Bibliográficas $\quad 45$ 


\section{Introdução}

O conceito de número de rotação de homeomorfismos do círculo $\mathbb{S}^{1}$ que são homotópicos à identidade foi introduzido por Henri Poincaré em [18] e tem tido uma história rica nos sistemas dinâmicos. Quando esse conceito é generalizado para a família de homeomorfismos homotópicos à identidade do toro $\mathbb{T}^{n}$, obtemos um subconjunto do espaço Euclidiano chamado conjunto de rotação. Existem várias noções deste conjunto para aplicações de $\mathbb{T}^{n}$, sendo mais usada a definição introduzida por Misiurewicz e Ziemian em [16].

Nas duas últimas décadas o conjunto de rotação tem sido amplamente explorado por vários matemáticos, entre eles, J. Franks [7], [9], M. Handel [10], [11], Libre e MacKay [15], Berge e Walker [2], Jonker e Zhang [12], além de Misiurewicz e Ziemian [16], [17]. Espera-se que o conjunto contenha informações a respeito da dinâmica do homeomorfismo e especialmente sobre a existência de pontos periódicos (como no caso de homeomorfismos do círculo). O que acontece é que no caso de um homeomorfismo $f$ do toro $\mathbb{T}^{2}$, o conjunto de rotação, denotado por $\rho(F)$ onde $F$ é um levantamento de $f$, tem "boas" propriedades e continua nos fornecendo informações sobre a dinâmica do homeomorfismo, embora essas informações não sejam tão completas como no caso do círculo. Já em dimensões maiores, no toro $\mathbb{T}^{n}$ com $n \geq 3$, o conjunto de rotação parece ser pouco útil até o momento.

Misiurewicz e Ziemian, em [16], mostraram que $\rho(F)$ é covexo. Franks mostrou em [7] que, se o conjunto de rotação possui um ponto racional com coordenadas $\left(p_{1} / n, p_{2} / n\right)$ em seu interior e $\operatorname{mdc}\left(p_{1}, p_{2}, n\right)=1$, então $f$ possui ponto periódico de período $n$.

Quando $\rho(F)$ é um segmento de reta (interior vazio), assumindo que $f$ preserva área, Franks mostrou em [9] que, se existe um vetor $v \in \rho(F)$ com coordenadas racionais, então $f$ tem ponto periódico.

O objetivo principal deste trabalho é demonstrar o teorema seguinte, obtido por Jonker e Zhang em [12], para o caso em que o conjunto de rotação é um segmento de reta com inclinação irracional: 
Teorema Principal Suponha que f é um homeomorfismo do toro $\mathbb{T}^{2}$ homotópico à identidade. Seja $F$ um levantamento de $f$ e suponha que o conjunto de rotação $\rho(F)$ é um segmento de reta com inclinação irracional. Se $\left(p_{1} / n, p_{2} / n\right) \in \rho(F), \operatorname{com} \operatorname{mdc}\left(p_{1}, p_{2}, n\right)=1$, então $f$ tem um ponto periódico de período $n$.

A seguir damos uma breve descrição de como a presente dissertação está organizada.

No Capítulo 1, veremos algumas definições e resultados importantes que serão utilizados posteriormente, o conceito de número de rotação para homeomorfismos de $\mathbb{S}^{1}$ e o Teorema de Poincaré, onde observaremos que "o número de rotação de um homeomorfismo $f$ de $\mathbb{S}^{1}$ homotópico à identidade é um racional $\frac{p}{q}, \operatorname{com} \operatorname{mdc}(p, q)=1$, se, e somente se, $f$ tem um ponto periódico de período q".

No Capítulo 2, definiremos o conjunto de rotação para homeomorfismos de $\mathbb{T}^{2}$ que são homotópicos à identidade e veremos propriedades importantes que são semelhantes ao caso dos homeomorfismos de $\mathbb{S}^{1}$, além dos resultados do trabalho de Conley sobre recorrência por cadeia em [4], a definição de função de Lyapounov completa e o Teorema de Conley. Apresentaremos também, um dos resultados fundamentais para a demonstração do Teorema Principal, a Proposição 2.3, onde concluímos que, "se $f$ não tem ponto periódico e o conjunto de rotação $\rho(F)$ é um segmento de reta com inclinação irracional, então $\mathbb{T}^{2}$ é transitivo por cadeia por $f^{\prime \prime}$.

O Capítulo 3 é dedicado à prova do Teorema Principal, dividindo-a em três casos. Quando o ponto racional $\left(p_{1} / n, p_{2} / n\right)$ é um extremo do conjunto de rotação, a conclusão segue imediatamente do Lema 2.1, que é um corolário dos Teoremas 2.4 e 3.10 do trabalho de Misiurewicz e Ziemian em [16], e diz o seguinte:

"Seja $F$ um levantamento do homeomorfismo $f: \mathbb{T}^{2} \rightarrow \mathbb{T}^{2}$ homotópico à identidade. Se $v \in \rho(F)$ é um ponto extremo, então existe um $x \in \mathbb{T}^{2} \operatorname{com} \rho(F, x)=v$. Se, além disso, $v$ é um ponto racional, digamos $v=\left(p_{1} / n, p_{2} / n\right)$ onde $\operatorname{mdc}\left(p_{1}, p_{2}, n\right)=1$, então $x$ pode ser escolhido de maneira que ele seja um ponto periódico com período $n$ ".

Para o caso em que o ponto racional não é um extremo, supondo que ele seja a origem, a existência de um ponto periódico é provada utilizando a Proposição 3.1 e o Lema 3.8, sendo que, a Proposição 3.1 é encontrada após construir um grafo orientado definido por meio de uma partição do toro $\mathbb{T}^{2}$, e o Lema 3.8 é um resultado do trabalho de Franks em [7].

Quando o ponto racional não é um extremo de $\rho(F)$ e não é a origem, através da 
Proposição 2.1, que mostra algumas propriedades do conjunto de rotação, nós voltamos ao caso anterior e a conclusão segue imediatamente. Finalmente, mostramos que o ponto periódico tem período $n$.

No final do Capítulo 3 nós damos um exemplo de um homeomorfismo do toro homotópico à identidade cujo conjunto de rotação é um segmento de reta com inclinação racional que contem infinitos pontos racionais mas que não possui nenhum ponto periódico. 


\section{Capítulo 1}

\section{Preliminares}

Veremos algumas definições e resultados importantes que serão utilizados posteriormente. Além do conceito de número de rotação para homeomorfismos do círculo que são homotópicos à identidade, algumas propriedades, e o Teorema 1.4 (Poincaré) que mostra a relação entre o número de rotação e a existência de pontos periódicos.

\subsection{Conceitos e resultados importantes}

Os resultados desta seção serão apenas enunciados. Maiores detalhes podem ser encontrados nas referências [14] e [19].

Dado um espaço métrico $X$ e uma função contínua $f: X \rightarrow X$, dizemos que o par $(X, f)$ é um sistema dinâmico e chamamos $X$ de espaço de fase.

Definição 1.1 Seja $(X, f)$ um sistema dinâmico.

1. Dado $x \in X, x$ é periódico se existe um $p \in \mathbb{N}$ tal que $f^{p}(x)=x$. Neste caso, se $f^{k}(x) \neq x$ para todo $k<p$, dizemos que $x$ tem período $p$. Se $f(x)=x, x$ é um ponto fixo para $f$.

2. Denotaremos o conjunto dos pontos periódicos de $f$ por $\operatorname{Per}(f)$, e o conjunto dos pontos fixos por Fix $(f)$.

3. A órbita do ponto $x \in X$ é o conjunto $\mathcal{O}_{f}(x)=\left\{f^{n}(x) ; n \geq 0\right\}$.

Teorema 1.1 (Ponto fixo de Brouwer) Seja B uma bola fechada de centro 0 e raio 1 em $\mathbb{R}^{n+1}$. Toda aplicação contínua $f: B \rightarrow B$ possui (pelo menos) um ponto fixo. 
O Teorema 1.2 obtido por Brouwer será utilizado na demonstração do Teorema Principal e pode ser encontrado nos trabalhos de Brown em [3] e Fathi em [6].

Teorema 1.2 (Brouwer) Se $f: \mathbb{R}^{2} \rightarrow \mathbb{R}^{2}$ é um homeomorfismo que preserva orientação e possui um ponto periódico, então f tem um ponto fixo.

Definição 1.2 Um grafo $G$ é um par $(V(G), E(G))$ onde $V(G)$ é um conjunto finito nãovazio cujos elementos são chamados de vértices, e $E(G)$ é um conjunto finito de pares distintos de elementos de $V(G)$, estes pares são chamados de arestas.

Um grafo $G$ é dito orientado quando o conjunto de arestas $E(G)$ é formado por pares ordenados.

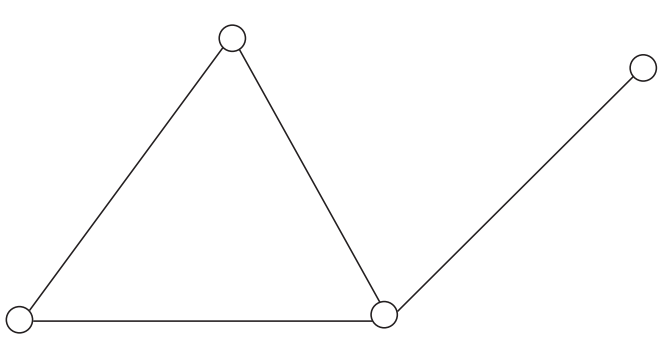

Figura 1.1: Grafo.

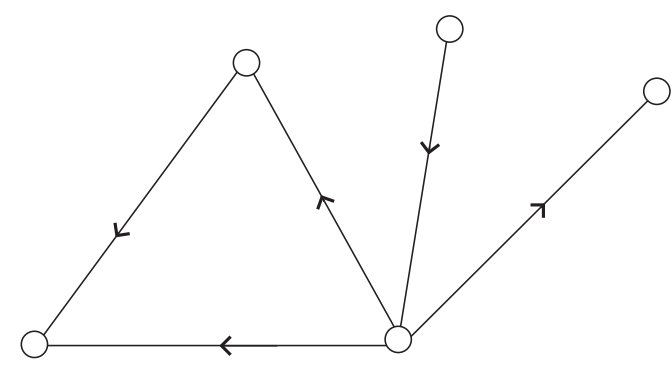

Figura 1.2: Grafo orientado.

Lema 1.1 Sejam $p=\left(p_{1}, p_{2}\right) \in \mathbb{Z}^{2}$ e $n \in \mathbb{N} \operatorname{com} \operatorname{mdc}\left(p_{1}, p_{2}, n\right)=1$. Se $h=\frac{k}{n} p \in \mathbb{Z}^{2}$ para algum $k \in \mathbb{N}$, então $k$ é múltiplo de $n$.

O seguinte teorema aparece na teoria de variedades diferenciáveis em contextos mais gerais.

Teorema 1.3 Sejam $U$ um aberto em $\mathbb{R}^{n}$ e $f: U \rightarrow \mathbb{R}^{n-m}$ de classe $C^{k}, k \geq 1$. Se $c \in \mathbb{R}^{n-m}$ é valor regular de $f$, então $f^{-1}(c)$ é uma sub-variedade de $\mathbb{R}^{n}$ de classe $C^{k} e$ dimensão $m$.

\subsection{Homeomorfismos do círculo e número de rotação}

Primeiro introduziremos a noção número de rotação para homeomorfismos do círculo que são homotópicos à identidade. Para isso precisaremos de alguns resultados sobre 
os levantamentos desses homeomorfismos. Finalmente, após estabelecer uma série de propriedades do número de rotação, mostramos o Teorema de Poincaré, Teorema 1.4. Para maiores detalhes podem ser consultadas as referências [5] e [13].

Denotaremos o círculo por $\mathbb{S}^{1}=\mathbb{R} / \mathbb{Z}$ e $\pi: \mathbb{R} \rightarrow \mathbb{S}^{1}$ a projeção natural, isto é, $\pi(x)=$ $x(\bmod 1)$. Um levantamento de um homeomorfismo $f: \mathbb{S}^{1} \rightarrow \mathbb{S}^{1}$ é um homeomorfismo $F: \mathbb{R} \rightarrow \mathbb{R}$ com a seguinte propriedade:

$$
f \circ \pi=\pi \circ F \text {. }
$$

Observação 1.1 (1) Se F e $G$ são dois levantamentos de $f$, então $F-G$ é uma aplicação constante de valor inteiro.

De fato, como $\pi \circ F=\pi \circ G$ temos que

$$
\pi \circ F(x)=\pi \circ G(x) \Rightarrow F(x)-G(x)=N(x) \in \mathbb{Z}, \forall x \in \mathbb{R}
$$

evidentemente $F-G$ é uma função contínua e portanto $F-G$ é constante.

(2) $f^{n} \circ \pi=\pi \circ F^{n}$, ou seja, $F^{n}$ é um levantamento de $f^{n}$ para qualquer $n \in \mathbb{N}$.

De fato,

$$
\begin{aligned}
f^{n} \circ \pi & =f^{n-1} \circ f \circ \pi \\
& =f^{n-1} \circ \pi \circ F \\
& =f^{n-2} \circ f \circ \pi \circ F \\
& =f^{n-2} \circ \pi \circ F^{2} \\
& \vdots \\
& =\pi \circ F^{n} .
\end{aligned}
$$

Lema 1.2 Seja $f: \mathbb{S}^{1} \rightarrow \mathbb{S}^{1}$ uma aplicação continua, se F é um levantamento de $f$, então $F(x+1)-F(x)$ é um inteiro que independe de $x$ e do levantamento.

Prova: Primeiro mostramos a independência em $x$. Sabemos que $\pi(F(x+1))=f(\pi(x+$ 1)) $=f(\pi(x))=\pi(F(x))$, ou seja, $F(x+1)-F(x)=Z(x) \in \mathbb{Z}$, e pela continuidade $F(x+1)-F(x)$ não depende de $x$. Finalmente mostramos a independência em relação ao levantamento. Se $G$ é outro levantamento de $f$ então $\pi(G(x))=f(\pi(x))=\pi(F(x))$, isso implica que $F(x)-G(x)=N(x) \in \mathbb{Z}$, e novamente pela continuidade $F-G$ é uma função constante, portanto $F(x+1)-G(x+1)=F(x)-G(x)$, com isso $F(x+1)-F(x)=$ $G(x+1)-G(x)$. 
Definição 1.3 Se $f: \mathbb{S}^{1} \rightarrow \mathbb{S}^{1}$ é uma aplicação contínua e $F$ é um levantamento de $f$, então $F(x+1)-F(x)$ é chamado de grau de $f$ e denotamos por $\operatorname{deg}(f)$.

Observação 1.2 Se $\operatorname{deg}(f)=1$, então $F(x+k)-F(x)=k$ para qualquer $k \in \mathbb{Z}$.

De fato, dado $k \in \mathbb{Z}_{+}$,

$$
\begin{aligned}
F(x+k) & =F(x+(k-1))+1 \\
& =F(x+(k-2))+2 \\
& \vdots \\
& =F(x)+k,
\end{aligned}
$$

ou seja, $F(x+k)-F(x)=k$.

No caso em que $k \in \mathbb{Z}_{-}$basta verificar que $F(x-1)-F(x)=-1$ e chegamos a $F(x+k)-F(x)=k$ de maneira análoga.

$$
\begin{aligned}
F(x-1)-F(x) & =F(x-1)-F((x-1)+1) \\
& =F(x-1)-(F(x-1)+1) \\
& =-1 .
\end{aligned}
$$

Proposição 1.1 Sejam $f, g: \mathbb{S}^{1} \rightarrow \mathbb{S}^{1}$ aplicações contínuas. Então $\operatorname{deg}(f)=\operatorname{deg}(g)$ se, e somente se, f e g são homotópicas.

A prova da proposição anterior pode ser encontrada em [13].

Corolário 1.1 Seja $f: \mathbb{S}^{1} \rightarrow \mathbb{S}^{1}$ continua. Então deg(f)=1 se, e somente se, $f$ é homotópica à identidade.

Proposição $1.2 \operatorname{deg}(f \circ g)=\operatorname{deg}(f) \cdot \operatorname{deg}(g)$.

Prova: Sejam $k=\operatorname{deg}(f), r=\operatorname{deg}(g)$ e $F, G$ levantamentos de $f$ e $g$ respectivamente, então $F \circ G$ é um levantamento de $f \circ g$ e

$$
\begin{aligned}
F \circ G(x+1) & =F(G(x+1)) \\
& =F(G(x)+r) \\
& =F(G(x))+k r \\
& =F \circ G(x)+k r .
\end{aligned}
$$

Isto conclui a prova. 
Corolário $1.2 \operatorname{deg}\left(f^{n}\right)=(\operatorname{deg}(f))^{n}, \operatorname{para} n \in \mathbb{N}$.

Proposição 1.3 Seja $f: \mathbb{S}^{1} \rightarrow \mathbb{S}^{1}$ um homeomorfismo homotópico à identidade. Se $F: \mathbb{R} \rightarrow \mathbb{R}$ é um levantamento de $f$, então $F$ é uma função crescente.

Prova: Como $f$ é homotópica à identidade, $\operatorname{deg}(f)=1$. Sendo $F: \mathbb{R} \rightarrow \mathbb{R}$ um homeomorfismo, $F$ é crescente ou decrescente. Escolhemos um levantamento $F$ de modo que $F(1) \in[0,1)$.

Se o grau de $f$ é 1 , então $F(x+1)-F(x)=1$ para todo $x \in \mathbb{R}$, e em particular, para $x=0$ e $x=-1$ temos

$$
\begin{gathered}
F(1)-F(0)=1 \\
F(0)-F(-1)=1 .
\end{gathered}
$$

Somando membro a membro as igualdades (1.1) e (1.2), obtemos

$$
F(1)-F(-1)=2 \Rightarrow F(1)=2+F(-1)
$$

mas $0 \leq F(1)<1$, segue que

$$
-2 \leq F(-1)<-1
$$

Daí, $F(-1)<F(1)$, e deste modo $F$ não pode ser decrescente, pois é um homeomorfismo de $\mathbb{R}$. Portanto, $F$ é crescente. Como qualquer levantamento de $f$ difere de $F$ apenas pela soma de um inteiro, concluímos que todo levantamento de $f$ é uma função crescente.

Proposição 1.4 Seja $f: \mathbb{S}^{1} \rightarrow \mathbb{S}^{1}$ um homeomorfismo homotópico à identidade e $F$ : $\mathbb{R} \rightarrow \mathbb{R}$ um levantamento de $f$. Então vale o seguinte:

(1) O limite $\tau(F):=\lim _{n \rightarrow \infty} \frac{F^{n}(x)-x}{n}$ existe para todo $x \in \mathbb{R}$;

(2) $\tau(F)$ não depende de $x$;

(3) Dados $F, G$ dois levantamentos de $f, \tau(F)-\tau(G)=F-G \in \mathbb{Z}$;

(4) Se f tem ponto periódico de período q então $\tau(F)=\frac{p}{q} \in \mathbb{Q}$ para algum $p \in \mathbb{Z}$.

Prova: (2) Como $f$ é um homeomorfismo homotópico à identidade $\operatorname{deg}(f)=1$, conseqüentemente, pelo Corolário $1.2, \operatorname{deg}\left(f^{n}\right)=1$ para qualquer $n \in \mathbb{N}$, ou seja, $F^{n}(x+1)=$ $F^{n}(x)+1$. Mais ainda, pela Proposição $1.3, F^{n}$ é crescente. Logo, se $x$ e $y$ são dois números reais tais que $|y-x|<1$, então 


$$
\begin{gathered}
y<x+1 \Rightarrow F^{n}(y)<F^{n}(x+1)=F^{n}(x)+1 \Rightarrow F^{n}(y)-F^{n}(x)<1 \\
x<y+1 \Rightarrow F^{n}(x)<F^{n}(y+1)=F^{n}(y)+1 \Rightarrow F^{n}(x)-F^{n}(y)<1, \\
\therefore\left|F^{n}(y)-F^{n}(x)\right|<1, \forall n \in \mathbb{N} .
\end{gathered}
$$

Daí,

$$
\begin{aligned}
\left|\frac{F^{n}(x)-x}{n}-\frac{F^{n}(y)-y}{n}\right| & =\frac{1}{n}\left|\left(F^{n}(x)-F^{n}(y)\right)+(y-x)\right| \\
& \leq \frac{1}{n}\left(\left|F^{n}(x)-F^{n}(y)\right|+|y-x|\right) \\
& <\frac{2}{n} .
\end{aligned}
$$

Deste modo, se o limite de $\frac{F^{n}(x)-x}{n}$ quando $n \rightarrow \infty$ existe, concluímos que

$$
\lim _{n \rightarrow \infty} \frac{F^{n}(x)-x}{n}=\lim _{n \rightarrow \infty} \frac{F^{n}(y)-y}{n}, \forall x, y \in \mathbb{R} \operatorname{com}|y-x|<1
$$

e portanto $\tau(F)=\lim _{n \rightarrow \infty} \frac{F^{n}(x)-x}{n}$ é o mesmo para qualquer $x \in \mathbb{R}$.

(4) Seja $\pi\left(x_{0}\right)$ um ponto periódico de $f$ de período $q$. Como $\pi\left(F^{q}\left(x_{0}\right)\right)=f^{q}\left(\pi\left(x_{0}\right)\right)=$ $\pi\left(x_{0}\right)$ temos que,

$$
F^{q}\left(x_{0}\right)=x_{0}+p
$$

para algum $p \in \mathbb{Z}$. Conseqüentemente,

$$
F^{m q}\left(x_{0}\right)=x_{0}+m p, \forall m \in \mathbb{Z}_{+}
$$

Mostraremos que $\tau(F)=\frac{p}{q}$. De fato, dado $n \in \mathbb{N}$, existem $m, i \in \mathbb{Z}_{+}$, satisfazendo $n=m q+i$, com $0 \leq i<q$. Daí, como $F^{n}\left(x_{0}\right)=F^{m q+i}\left(x_{0}\right)=F^{i}\left(F^{m q}\left(x_{0}\right)\right)=F^{i}\left(x_{0}+\right.$ $m p)=F^{i}\left(x_{0}\right)+m p$, temos que

$$
\frac{F^{n}\left(x_{0}\right)-x_{0}}{n}=\frac{F^{i}\left(x_{0}\right)+m p-x_{0}}{m q+i}=\frac{F^{i}\left(x_{0}\right)-x_{0}}{m q+i}+\frac{m p}{m q+i} .
$$

Portanto,

$$
\tau(F)=\lim _{n \rightarrow \infty} \frac{F^{n}\left(x_{0}\right)-x_{0}}{n}=\frac{p}{q} .
$$

(1) Pelo que vimos em (2) basta mostrar que o limite existe para $x=0$. Do item (4) sabemos que se $f$ tem ponto periódico, então existe $\tau(F)$. Sendo assim, suponhamos que 
$f$ não tem ponto periódico, com isso $F^{m}(x)-x \notin \mathbb{Z}$ para todo $m \in \mathbb{N}$ e qualquer $x$ real. Em particular, tomando $x=0$, para cada $m \in \mathbb{N}$ existe um $h_{m} \in \mathbb{Z}$ tal que

$$
h_{m}<F^{m}(0)<h_{m}+1 \text {. }
$$

Conseqüentemente, para $r \in \mathbb{Z}_{+}$arbitrário, temos:

$$
\begin{gathered}
F^{r}\left(h_{m}\right)<F^{r}\left(F^{m}(0)\right)<F^{r}\left(h_{m}+1\right) \\
F^{r}(0)+h_{m}<F^{r+m}(0)<F^{r}(0)+h_{m}+1 \\
h_{m}<F^{r+m}(0)-F^{r}(0)<h_{m}+1 .
\end{gathered}
$$

Dado $n \in \mathbb{N}$ e tomando $r=0, m, 2 m, \ldots,(n-1) m$ obtemos as desigualdades

$$
\begin{gathered}
h_{m}<F^{m}(0)<h_{m}+1 \\
h_{m}<F^{2 m}(0)-F^{m}(0)<h_{m}+1 \\
\vdots \\
h_{m}<F^{n m}(0)-F^{(n-1) m}(0)<h_{m}+1 .
\end{gathered}
$$

Somando estas desigualdades membro a membro, obtemos

$$
n h_{m}<F^{n m}(0)<n\left(h_{m}+1\right) .
$$

Logo,

$$
\frac{h_{m}}{m}<\frac{F^{n m}(0)}{n m}<\frac{h_{m}+1}{m}
$$

em particular para $n=1$,

$$
\frac{h_{m}}{m}<\frac{F^{m}(0)}{m}<\frac{h_{m}+1}{m} .
$$

Daí concluímos que

$$
\left|\frac{F^{n m}(0)}{n m}-\frac{F^{m}(0)}{m}\right|<\frac{1}{m} .
$$

De maneira análoga trocando $n$ por $m$ e $m$ por $n$ também temos

$$
\left|\frac{F^{n m}(0)}{n m}-\frac{F^{n}(0)}{n}\right|<\frac{1}{n} \text {. }
$$

Destas duas últimas desigualdades obtemos

$$
\left|\frac{F^{m}(0)}{m}-\frac{F^{n}(0)}{n}\right| \leq\left|\frac{F^{m}(0)}{m}-\frac{F^{n m}(0)}{n m}\right|+\left|\frac{F^{n m}(0)}{n m}-\frac{F^{n}(0)}{n}\right|<\frac{1}{m}+\frac{1}{n} .
$$


Isto significa que a seqüência $\left\{\frac{F^{n}(0)}{n}\right\}_{n \in \mathbb{N}}$ é de Cauchy, e portanto o limite $\lim _{n \rightarrow \infty} \frac{F^{n}(0)}{n}$ existe.

(3) Dados $F$ e $G$ dois levantamentos de $f$, existe $k \in \mathbb{Z}$ tal que $F-G=k$. Daí,

$$
\begin{aligned}
\tau(F) & =\lim _{n \rightarrow \infty} \frac{F^{n}(x)-x}{n} \\
& =\lim _{n \rightarrow \infty} \frac{(G+k)^{n}(x)-x}{n} \\
& =\lim _{n \rightarrow \infty} \frac{G^{n}(x)-x+n k}{n} \\
& =\left[\lim _{n \rightarrow \infty} \frac{G^{n}(x)-x}{n}\right]+k \\
& =\tau(G)+k .
\end{aligned}
$$

Ao limite $\tau(F)$ nós chamamos de número de translação de $F$, e o resultado anterior nos permite fazer a seguinte definição:

Definição $1.4 \rho(f):=\pi(\tau(F))$ é chamado de número de rotação de $f$.

Exemplo: Vejamos os tipos mais simples de homeomorfismos de $\mathbb{S}^{1}$ que são homotópicos à identidade, as rotações. Se $\alpha \in[0,1)$, definimos $R_{\alpha}$ a rotação em $\mathbb{S}^{1}$ como:

$$
R_{\alpha}(\theta)=\theta+\alpha(\bmod 1) .
$$

A órbita de um ponto $\theta_{0}$ pela rotação $R_{\alpha}$ é

$$
\mathcal{O}_{R_{\alpha}}\left(\theta_{0}\right)=\left\{\theta_{0}+n \alpha: n \geq 0\right\}
$$

Se $\alpha$ é um número racional, digamos $\alpha=p / q$ para $p$ e $q$ inteiros primos entre si, então $q \alpha \in \mathbb{Z}$, e neste caso a órbita $\mathcal{O}_{R_{\frac{p}{q}}}\left(\theta_{0}\right)=\left\{\theta_{0}+n \frac{p}{q}: n \geq 0\right\}$ é periódica de período $q$ para qualquer $\theta_{0}$. Quando $\alpha$ é irracional, pode ser mostrado que não existem órbitas periódicas, mais ainda, a órbita de qualquer ponto é densa em $\mathbb{S}^{1}$. Em ambos os casos $\rho\left(R_{\alpha}\right)=\alpha$.

Para qualquer homeomorfismo do círculo homotópico à identidade Poincaré demonstrou o seguinte teorema:

Teorema 1.4 (Poincaré) Seja $f: \mathbb{S}^{1} \rightarrow \mathbb{S}^{1}$ um homeomorfismo homotópico à identidade. Então o número de rotação $\rho(f)=\frac{p}{q} \in \mathbb{Q}$, com $\operatorname{mdc}(p, q)=1$, se, e somente se, a aplicação f tem um ponto periódico de período q. 
Prova: Pela Proposição 1.4, se $f$ tem um ponto periódico de período $q$, então $\rho(f)=\frac{p}{q}$. Suponhamos agora que $\rho(f)=\frac{p}{q} \in \mathbb{Q}$. Mostraremos que $f$ tem ponto periódico de período $q$.

$$
\begin{aligned}
\rho\left(f^{m}\right) & =\lim _{n \rightarrow \infty} \frac{\left(F^{m}\right)^{n}(x)-x}{n}(\bmod 1) \\
& =m \lim _{n \rightarrow \infty} \frac{F^{m n}(x)-x}{m n}(\bmod 1) \\
& =m \rho(f)(\bmod 1),
\end{aligned}
$$

para $m \in \mathbb{N}$. Então

$$
\rho\left(f^{q}\right)=p(\bmod 1)=0 .
$$

Logo, é suficiente mostrar que $f^{q}$ tem ponto fixo.

Suponhamos que $f^{q}$ não tem ponto fixo, conseqüentemente $F^{q}(x)-x \notin \mathbb{Z}$ para qualquer $x$ real. Escolhemos o levantamento $F$ de maneira que $F^{q}(0) \in(0,1)$. Com isso, pela continuidade de $F^{q}-i d_{\mathbb{R}}$, temos que $0<F^{q}(x)-x<1$ para todo $x \in \mathbb{R}$. Deste modo, como $\left(F^{q}-i d_{\mathbb{R}}\right)([0,1])=\left(F^{q}-i d_{\mathbb{R}}\right)(\mathbb{R})$, existe $\delta>0$ tal que

$$
0<\delta \leq F^{q}(x)-x \leq 1-\delta<1, \forall x \in \mathbb{R}
$$

Dado $r \in \mathbb{Z}_{+}$qualquer, e fazendo $x=F^{r}(0)$,

$$
\begin{aligned}
\delta \leq F^{q}(x)-x \leq 1-\delta & \Rightarrow \delta \leq F^{q}\left(F^{r}(0)\right)-F^{r}(0) \leq 1-\delta \\
& \Rightarrow \delta \leq F^{q+r}(0)-F^{r}(0) \leq 1-\delta .
\end{aligned}
$$

Tomando $r=0, q, 2 q, \ldots,(n-1) q$ para $n \in \mathbb{N}$,

$$
\begin{gathered}
\delta \leq F^{q}(0) \leq 1-\delta \\
\delta \leq F^{2 q}(0)-F^{q}(0) \leq 1-\delta \\
\vdots \\
\delta \leq F^{n q}(0)-F^{(n-1) q}(0) \leq 1-\delta
\end{gathered}
$$

somando todos os termos,

$$
\begin{aligned}
n \delta \leq F^{n q}(0) \leq n(1-\delta) & \Rightarrow n \delta \leq\left(F^{q}\right)^{n}(0) \leq n(1-\delta) \\
& \Rightarrow 0<\delta \leq \frac{\left(F^{q}\right)^{n}(0)}{n} \leq(1-\delta)<1
\end{aligned}
$$

e portanto $\rho\left(f^{q}\right)>0$, o que é uma contradição. Então $f^{q}$ tem ponto fixo. E assim concluímos que $f$ tem ponto periódico de período $q$. 


\section{Capítulo 2}

\section{Conjunto de rotação e transitividade por cadeias}

Na Seção 2.1 definiremos o conjunto de rotação (Definição 2.1) para homeomorfismos do toro $\mathbb{T}^{2}$ que são homotópicos à identidade e veremos propriedades importantes que são semelhantes ao caso dos homeomorfismos de $\mathbb{S}^{1}$.

Em seguida, na Seção 2.2, exploramos os resultados do trabalho de Conley em [4] sobre recorrência por cadeia, a definição de função de Lyapounov completa e o Teorema de Conley. Tudo isso para mostrar o resultado principal deste capítulo que é a Proposição 2.3, que estabelece o seguinte:

"Se $f$ não tem ponto periódico e o conjunto de rotação $\rho(F)$ é um segmento de reta com inclinação irracional, então o toro $\mathbb{T}^{2}$ é transitivo por cadeia por $f$ ".

\subsection{Conjunto de rotação: propriedades gerais}

Sejam $f: \mathbb{T}^{2} \rightarrow \mathbb{T}^{2}$ um homeomorfismo homotópico à identidade e $\pi: \mathbb{R}^{2} \rightarrow \mathbb{T}^{2}$ a aplicação de recobrimento. Um levantamento de $f$ é um homeomorfismo $F: \mathbb{R}^{2} \rightarrow \mathbb{R}^{2}$ tal que

$$
f \circ \pi=\pi \circ F \text {. }
$$

Com essas condições definimos o conjunto de rotação.

Definição 2.1 O conjunto de rotação $\rho(F)$ é o conjunto de todos os pontos $v \in \mathbb{R}^{2}$ para 
os quais existem seqüências $\left\{\widetilde{x}_{i}\right\} \subset \mathbb{R}^{2}$ e $\left\{n_{i}\right\} \subset \mathbb{N}$ com $n_{i} \rightarrow \infty$ quando $i \rightarrow \infty$ tais que

$$
\lim _{i \rightarrow \infty} \frac{F^{n_{i}}\left(\widetilde{x}_{i}\right)-\widetilde{x}_{i}}{n_{i}}=v
$$

Se o limite

$$
\lim _{n \rightarrow \infty} \frac{F^{n}(\widetilde{x})-\widetilde{x}}{n}
$$

existe, para $\widetilde{x} \in \pi^{-1}(x)$, nós o chamamos de vetor rotação de $x \in \mathbb{T}^{2}$ e denotamos este limite por $\rho(F, x)$.

O levantamento $F$ tem propriedades parecidas com as que observamos para homeomorfismos de $\mathbb{S}^{1}$ na Seção 1.2 .

Observação 2.1 $\quad$ (1) Se F e G são dois levantamentos de $f$, então $F-G$ é uma aplicação constante e $F(x)-G(x)=h$, para algum $h \in \mathbb{Z}^{2}$;

(2) Além disso, para quaisquer $x \in \mathbb{R}^{2}$ e $p \in \mathbb{Z}^{2}$,

$$
F(x+p)=F(x)+p
$$

O conjunto de rotação não depende apenas de $f$, se substituímos $F$ por outro levantamento $G, \rho(G)$ é a translação de $\rho(F)$ por um ponto de $\mathbb{Z}^{2}$. Por esta razão nós escrevemos $\rho(F)$ e não $\rho(f)$. A Proposição 2.1 confirma esta afirmação e mostra a relação que existe entre $\rho\left(F^{n}\right)$ e $\rho(F)$.

Proposição 2.1 Dado $n \in \mathbb{N}$ e $p \in \mathbb{Z}^{2}$,

(1) $\rho(F+p)=\rho(F)+p$;

(2) $\rho\left(F^{n}\right)=n \rho(F)$.

Prova: (1) Sejam $\left\{\widetilde{x}_{i}\right\}_{i \in \mathbb{N}} \subset \mathbb{R}^{2}$ e $\left\{m_{i}\right\}_{i \in \mathbb{N}} \subset \mathbb{N}$ com $m_{i} \rightarrow \infty$ quando $i \rightarrow \infty$. Então

$$
\begin{aligned}
v=\lim _{i \rightarrow \infty} \frac{F^{m_{i}}\left(\widetilde{x}_{i}\right)-\widetilde{x}_{i}}{m_{i}} \Leftrightarrow v+p & =\lim _{i \rightarrow \infty} \frac{F^{m_{i}}\left(\widetilde{x}_{i}\right)-\widetilde{x}_{i}}{m_{i}}+p \\
\Leftrightarrow v+p & =\lim _{i \rightarrow \infty} \frac{F^{m_{i}}\left(\widetilde{x}_{i}\right)+m_{i} p-\widetilde{x}_{i}}{m_{i}} \\
\Leftrightarrow v+p & =\lim _{i \rightarrow \infty} \frac{(F+p)^{m_{i}}\left(\widetilde{x}_{i}\right)-\widetilde{x}_{i}}{m_{i}} .
\end{aligned}
$$

Conseqüentemente,

$$
v \in \rho(F) \Longleftrightarrow v+p \in \rho(F+p) .
$$


Isto prova (1).

(2) $\rho\left(F^{n}\right) \subset n \rho(F)$ : Se $v \in \rho\left(F^{n}\right)$ então existem $\left\{\widetilde{x}_{i}\right\}_{i \in \mathbb{N}} \subset \mathbb{R}^{2}$ e $\left\{m_{i}\right\}_{i \in \mathbb{N}} \subset \mathbb{N}$ com $m_{i} \rightarrow \infty$ quando $i \rightarrow \infty$ tais que

$$
v=\lim _{i \rightarrow \infty} \frac{\left(F^{n}\right)^{m_{i}}\left(\widetilde{x}_{i}\right)-\widetilde{x}_{i}}{m_{i}}=\lim _{i \rightarrow \infty} \frac{F^{n m_{i}}\left(\widetilde{x}_{i}\right)-\widetilde{x}_{i}}{m_{i}}
$$

isto implica que

$$
\frac{1}{n} v=\lim _{i \rightarrow \infty} \frac{F^{n m_{i}}\left(\widetilde{x}_{i}\right)-\widetilde{x}_{i}}{n m_{i}}
$$

Conseqüentemente, $\frac{1}{n} v \in \rho(F)$. Assim, $\rho\left(F^{n}\right) \subset n \rho(F)$.

(2) $n \rho(F) \subset \rho\left(F^{n}\right)$ : Se $v \in \rho(F)$, então existem $\left\{\widetilde{x}_{i}\right\}_{i \in \mathbb{N}} \subset \mathbb{R}^{2}$ e $\left\{m_{i}\right\}_{i \in \mathbb{N}} \subset \mathbb{N}$ tais que $m_{i} \rightarrow \infty$ quando $i \rightarrow \infty$ e

$$
\lim _{i \rightarrow \infty} \frac{F^{m_{i}}\left(\widetilde{x}_{i}\right)-\widetilde{x}_{i}}{m_{i}}=v
$$

Dado $r \in \mathbb{Z}_{+}$, como $F^{r}-i d_{\mathbb{R}^{2}}$ é limitada em $[0,1] \times[0,1]$ e para cada $z \in \mathbb{R}^{2}$ existem $y \in[0,1] \times[0,1]$ e $h \in \mathbb{Z}^{2}$ tais que $z=y+h$, então existe um $M_{r}>0$ tal que

$$
\left\|F^{r}(z)-z\right\| \leq M_{r}, \forall z \in \mathbb{R}^{2}
$$

Mais ainda, tomando $M:=\max \left\{M_{r} ; 0 \leq r \leq n-1\right\}$, obtemos

$$
\left\|F^{r}(z)-z\right\| \leq M, \forall z \in \mathbb{R}^{2} \text { e } \forall r \in \mathbb{Z} \operatorname{com} 0 \leq r \leq n-1
$$

Vamos mostrar que existe $\left\{k_{i}\right\} \subset \mathbb{N}$ com $k_{i} \rightarrow \infty$ quando $i \rightarrow \infty$ tal que $n v \in \rho\left(F^{n}\right)$. Isto mostrará que $n \rho(F) \subset \rho\left(F^{n}\right)$.

Pelo algoritmo da divisão, para $m_{i} \geq n$ existem $\left\{k_{i}\right\} \subset \mathbb{N}$ e $\left\{r_{i}\right\} \subset \mathbb{Z}$ com $0 \leq r_{i} \leq n-1$ tais que

$$
m_{i}=n k_{i}+r_{i}
$$

Afirmamos que $v=\lim _{i \rightarrow \infty} \frac{F^{n k_{i}}\left(\widetilde{x}_{i}\right)-\widetilde{x}_{i}}{n k_{i}}$. De fato, fazendo $z_{i}=F^{n k_{i}}\left(\widetilde{x}_{i}\right)\left(\widetilde{x}_{i}=F^{-n k_{i}}\left(z_{i}\right)\right)$,

$$
\begin{gathered}
\left\|\frac{F^{m_{i}}\left(\widetilde{x}_{i}\right)-\widetilde{x}_{i}}{m_{i}}-\frac{F^{n k_{i}}\left(\widetilde{x}_{i}\right)-\widetilde{x}_{i}}{n k_{i}}\right\| \leq \\
\left\|\frac{F^{m_{i}}\left(\widetilde{x}_{i}\right)-\widetilde{x}_{i}}{m_{i}}-\frac{F^{m_{i}}\left(\widetilde{x}_{i}\right)-\widetilde{x}_{i}}{n k_{i}}\right\|+\left\|\frac{F^{m_{i}}\left(\widetilde{x}_{i}\right)-\widetilde{x}_{i}}{n k_{i}}-\frac{F^{n k_{i}}\left(\widetilde{x}_{i}\right)-\widetilde{x}_{i}}{n k_{i}}\right\|= \\
\left\|\frac{\left(n k_{i}-m_{i}\right)\left(F^{m_{i}}\left(\widetilde{x}_{i}\right)-\widetilde{x}_{i}\right)}{m_{i} n k_{i}}\right\|+\left\|\frac{F^{m_{i}}\left(\widetilde{x}_{i}\right)-F^{n k_{i}}\left(\widetilde{x}_{i}\right)}{n k_{i}}\right\|= \\
\left\|\frac{-r_{i}\left(F^{m_{i}}\left(\widetilde{x}_{i}\right)-\widetilde{x}_{i}\right)}{m_{i} n k_{i}}\right\|+\left\|F^{m_{i}-n k_{i}}\left(z_{i}\right)-z_{i}\right\| \frac{1}{n k_{i}}=
\end{gathered}
$$




$$
\left\|\frac{F^{m_{i}}\left(\widetilde{x}_{i}\right)-\widetilde{x}_{i}}{m_{i}}\right\| \frac{r_{i}}{n k_{i}}+\left\|F^{r_{i}}\left(z_{i}\right)-z_{i}\right\| \frac{1}{n k_{i}} .
$$

Mas, como $\left\|\frac{F^{m_{i}}\left(\widetilde{x}_{i}\right)-\widetilde{x}_{i}}{m_{i}}\right\| \rightarrow\|v\|$ e $\left\|F^{r}\left(z_{i}\right)-z_{i}\right\| \leq M$, então

$$
\left\|\frac{F^{m_{i}}\left(\widetilde{x}_{i}\right)-\widetilde{x}_{i}}{m_{i}}-\frac{F^{n k_{i}}\left(\widetilde{x}_{i}\right)-\widetilde{x}_{i}}{n k_{i}}\right\| \longrightarrow 0 \text { quando } i \rightarrow \infty,
$$

ou seja,

$$
v=\lim _{i \rightarrow \infty} \frac{F^{n k_{i}}\left(\widetilde{x}_{i}\right)-\widetilde{x}_{i}}{n k_{i}}=\frac{1}{n} \lim _{i \rightarrow \infty} \frac{\left(F^{n}\right)^{k_{i}}\left(\widetilde{x}_{i}\right)-\widetilde{x}_{i}}{k_{i}}
$$

Isto significa que

$$
n v=\lim _{i \rightarrow \infty} \frac{\left(F^{n}\right)^{k_{i}}\left(\widetilde{x}_{i}\right)-\widetilde{x}_{i}}{k_{i}} \Rightarrow n v \in \rho\left(F^{n}\right)
$$

Portanto,

$$
n \rho(F) \subset \rho\left(F^{n}\right)
$$

O teorema seguinte foi obtido por Misiurewicz e Ziemian em [16].

Teorema $2.1 \rho(F)$ é fechado, conexo e convexo.

Em particular, se $\rho(F)$ tem interior vazio, então $\rho(F)$ é um ponto ou um segmento de reta.

\subsection{Recorrência e transitividade por cadeias}

Nesta seção introduzimos três noções muito importantes, recorrência, transitividade por cadeia (Definição 2.2) e função de Lyapounov completa (Definição 2.3), além do teorema obtido por Conley em [4] sobre a existência dessa função.

O resultado principal desta seção é a Proposição 2.3, a qual garante que, "se $f$ não tem ponto periódico e o conjunto de rotação $\rho(F)$ é um segmento de reta com inclinação irracional, então o toro $\mathbb{T}^{2}$ é transitivo por cadeia por $f^{\prime \prime}$.

Definição 2.2 Sejam $(X, d)$ um espaço métrico compacto e $f: X \rightarrow X$ um homeomorfismo. Uma $\varepsilon$-cadeia de $x_{0}$ a $x_{n}$ para $f$ é uma seqüência finita de pontos $\left\{x_{i}\right\}_{i=0}^{n}$ em $X$, tal que $d\left(f\left(x_{i}\right), x_{i+1}\right)<\varepsilon$ para $0 \leq i<n$. Se $x_{0}=x_{n}$, dizemos que a $\varepsilon$-cadeia é periódica. Um ponto $x$ é dito recorrente por cadeia se para todo $\varepsilon>0$ existe um $\varepsilon$-cadeia de $x$ a 
$x$. Denotamos por $R(f)$ o conjunto de todos os pontos recorrentes por cadeia por $f$. Um subconjunto $\Lambda \subset X$ é chamado transitivo por cadeia por $f$ se para todo $\varepsilon>0$ e qualquer $x, y \in \Lambda$, existe uma $\varepsilon$-cadeia de $x$ a $y$.

Proposição 2.2 Seja $f: X \rightarrow X$. O conjunto dos pontos recorrentes por cadeia por $f$ é fechado e $f(R(f))=R(f)$.

Prova: (i) $R(f)$ é fechado: Provaremos que $X-R(f)$ é aberto. Se $\widetilde{x} \in X-R(f)$ então existe $\varepsilon>0$ tal que não existe nenhuma $\varepsilon$-cadeia de $\widetilde{x}$ a $\widetilde{x}$. Como $X$ é compacto, a continuidade de $f$ é uniforme, e portanto para $\varepsilon / 3>0$ podemos encontrar um $\delta>0$ de modo que, para quaisquer $x, y \in X$

$$
d(x, y)<\delta \Rightarrow d(f(x), f(y))<\varepsilon / 3
$$

Definimos $r:=\min \{\varepsilon / 3, \delta\}$. Mostraremos que $\widetilde{x}$ está no interior de $X-R(f)$, ou seja, dado $\widetilde{y} \in X \operatorname{com} d(\widetilde{x}, \widetilde{y})<r$, veremos que $\widetilde{y} \in X-R(f)$. Para isso, suponhamos por absurdo que $\widetilde{y}$ é recorrente por cadeia. Deste modo, existe uma seqüência finita $\left\{\widetilde{y}_{i}\right\}_{i=0}^{n} \operatorname{com} \widetilde{y}_{0}=\widetilde{y}_{n}=\widetilde{y}$ e $d\left(f\left(\widetilde{y}_{i}\right), \widetilde{y}_{i+1}\right)<r$ para $0 \leq i<n$. Escolhemos uma seqüência de pontos $\left\{\widetilde{x}_{i}\right\}_{i=0}^{n} \operatorname{com} \widetilde{x}_{0}=\widetilde{x}_{n}=\widetilde{x}$ tal que $d\left(\widetilde{x}_{i}, \widetilde{y}_{i}\right)<r \leq \delta$. De (2.1) temos que $d\left(f\left(\widetilde{x}_{i}\right), f\left(\widetilde{y}_{i}\right)\right)<\varepsilon / 3$ para $0 \leq i<n$, conseqüentemente

$$
\begin{aligned}
d\left(f\left(\widetilde{x}_{i}\right), \widetilde{x}_{i+1}\right) & \leq d\left(f\left(\widetilde{x}_{i}\right), f\left(\widetilde{y}_{i}\right)\right)+d\left(f\left(\widetilde{y}_{i}\right), \widetilde{y}_{i+1}\right)+d\left(\widetilde{y}_{i+1}, \widetilde{x}_{i+1}\right) \\
& <\varepsilon / 3+2 r \\
& <\varepsilon,
\end{aligned}
$$

contradizendo o fato de não existir uma $\varepsilon$-cadeia de $\widetilde{x}$ a $\widetilde{x}$. Isto mostra que $X-R(f)$ é aberto.

(ii) $f(R(f)) \subset R(f)$ : Dado $\widetilde{y} \in f(R(f)), \widetilde{y}=f(\widetilde{x})$ para algum $\widetilde{x} \in R(f)$. Seja $\varepsilon>0$ qualquer, pela continuidade uniforme de $f$, existe um $\delta>0$ tal que para qualquer $x, y \in X$

$$
d(x, y)<\delta \Rightarrow d(f(x), f(y))<\varepsilon .
$$

Como $\widetilde{x}$ é recorrente por cadeia por $f$, encontramos uma seqüência $\left\{\widetilde{x}_{i}\right\}_{i=0}^{n} \operatorname{com} \widetilde{x}_{0}=\widetilde{x}_{n}=$ $\widetilde{x}$ e $d\left(f\left(\widetilde{x}_{i}\right), \widetilde{x}_{i+1}\right)<\delta$ para $0 \leq i<n$. De $(2.2)$ segue que

$$
d\left(f\left(f\left(\widetilde{x}_{i}\right)\right), f\left(\widetilde{x}_{i+1}\right)\right)<\varepsilon, \forall 0 \leq i<n .
$$


Definindo $\widetilde{y}_{i}=f\left(\widetilde{x}_{i}\right)$ para $0 \leq i \leq n$ temos que, $\widetilde{y}_{0}=\widetilde{y}_{n}=\widetilde{y}$ e $d\left(f\left(\widetilde{y}_{i}\right), \widetilde{y}_{i+1}\right)<\varepsilon$ para qualquer $0 \leq i<n$, ou seja, $\widetilde{y}$ é recorrente por cadeia por $f$.

$$
\therefore f(R(f)) \subset R(f) \text {. }
$$

(iii) $R(f) \subset f(R(f))$ : Dado $x \in R(f)$ e um $\varepsilon>0$ qualquer, seja $\left\{x_{i}\right\}_{i=0}^{n} \operatorname{com} x_{0}=x_{n}=x$ e $d\left(f\left(x_{i}\right), x_{i+1}\right)<\varepsilon$ para $0 \leq i<n$.

Definimos $y_{i}:=f\left(x_{n-i}\right)$, deste modo $y_{0}=f\left(x_{n}\right)=y=f\left(x_{0}\right)=y_{n}$. Para $0 \leq i<n$

$$
d\left(f^{-1}\left(y_{i}\right), y_{i+1}\right)=d\left(f^{-1}\left(f\left(x_{n-i}\right)\right), f\left(x_{n-i-1}\right)\right)=d\left(x_{n-i}, f\left(x_{n-i-1}\right)\right)<\varepsilon
$$

isto implica que $y=f(x)$ é recorrente por cadeia por $f^{-1}$, ou seja

$$
f(R(f)) \subset R\left(f^{-1}\right)
$$

Equivalentemente,

$$
R(f) \subset f^{-1}\left(R\left(f^{-1}\right)\right) .
$$

Já sabemos de (ii) que $f(R(f)) \subset R(f)$ e utilizando este resultado para $f^{-1}$ obtemos

$$
f^{-1}\left(R\left(f^{-1}\right)\right) \subset R\left(f^{-1}\right)
$$

Usando o fato de $f(R(f)) \subset R\left(f^{-1}\right)$, para $f^{-1}$ também temos $f^{-1}\left(R\left(f^{-1}\right)\right) \subset R(f)$ e aplicando $f$ chegamos a

$$
R\left(f^{-1}\right) \subset f(R(f))
$$

De (2.3), (2.4) e (2.5) concluímos que

$$
R(f) \subset f(R(f))
$$

O que completa a prova.

Vamos definir uma relação de equivalência $\sim$ no conjunto $R(f)$. Dizemos que $x \sim y$ se, e somente se, para todo $\varepsilon>0$ existe uma $\varepsilon$-cadeia em $R(f)$ de $x$ a $y$ e também existe uma $\varepsilon$-cadeia em $R(f)$ de $y$ a $x$. As classes de equivalência em $R(f)$ determinadas pela relação de equivalência $\sim$ são chamadas de componentes transitivas por cadeia de $R(f)$.

Definição 2.3 Seja $f: X \rightarrow X$ um homeomorfismo de um espaço métrico compacto. Uma função de Lyapounov completa para $f$ é uma função contínua $g: X \rightarrow \mathbb{R}$ satisfazendo: 
(1) se $x \notin R(f)$, então $g(f(x))<g(x)$;

(2) para $x, y \in R(f), g(x)=g(y)$ se, e somente se, $x$ e $y$ estão na mesma componente transitiva por cadeia;

(3) $g(R(f))$ é um subconjunto compacto nunca-denso de $\mathbb{R}$.

Teorema 2.2 (Conley) Seja f um homeomorfismo de um espaço métrico compacto, então existe uma função de Lyapounov completa para $f$.

A Proposição 2.3 será fundamental na prova do Teorema Principal, e para fazer a demonstração dessa proposição utilizaremos o Lema 2.1, que é um corolário dos Teoremas 2.4 e 3.10 do trabalho de Misiurewicz e Ziemian em [16], e algumas definições e resultados encontrados em [7], em particular o Teorema 2.3, que apresentaremos a partir de agora.

Lema 2.1 Seja $F$ um levantamento do homeomorfismo $f: \mathbb{T}^{2} \rightarrow \mathbb{T}^{2}$ homotópico à identidade. Se $v \in \rho(F)$ é um ponto extremo, então existe um $x \in \mathbb{T}^{2} \operatorname{com} \rho(F, x)=v$. Se, além disso, $v$ é um ponto racional, digamos $v=\left(p_{1} / n, p_{2} / n\right)$ onde $\operatorname{mdc}\left(p_{1}, p_{2}, n\right)=1$, então $x$ pode ser escolhido de maneira que ele seja um ponto periódico com período $n$.

Em geral o número de componentes transitivas por cadeia pode ser infinito. Entretanto, se nós escolhemos um $\delta>0$ fixo e trabalhamos com as $\delta$-cadeias, podemos decompor $R(f)$ em um número finito de partes.

Definição 2.4 Dado $\delta>0$ fixo, dizemos que $x, y \in R(f)$ são $\delta$-equivalentes se existe uma $\delta$-cadeia de $x$ a $y$ e outra de $y$ a $x$. Esta é uma relação de equivalência e as classes de equivalência são chamadas de componentes $\delta$-transitivas de $R(f)$.

Lema 2.2 Dado $\delta>0$ e um homeomorfismo $f: X \rightarrow X$ de um espaço métrico compacto $X$, o número de componentes $\delta$-transitivas é finito.

Teorema 2.3 (Franks, [7]) Dado $\delta>0$ e um homeomorfismo de um espaço métrico compacto $f: X \rightarrow X$, existe uma função de Lyapounov completa $g: X \rightarrow \mathbb{R}$ para $f$, e $c_{0}<c_{1}<c_{2}<\ldots<c_{n}$ com $c_{i} \notin g(R(f))$ tal que, se $\Lambda_{i}=R(f) \cap g^{-1}\left(\left[c_{i-1}, c_{i}\right]\right)$, então $\left\{\Lambda_{i}\right\}$ com $1 \leq i \leq n$, são as componentes $\delta$-transitivas de $f$. 
Observação 2.2 (1) Pela construção da função de Lyapounov completa feita por Franks em [8] e [7], temos que

$$
g(f(x)) \leq g(x), \forall x \in X
$$

(2) Se $r \subset \mathbb{R}^{2}$ é uma reta com inclinação (coeficiente angular) irracional, então $\pi(r)$ é um subconjunto denso de $\mathbb{T}^{2}$.

De fato, seja $r \subset \mathbb{R}^{2}$ uma reta com equação $y=\alpha x$, sendo $\alpha$ irracional. Utilizando $[0,1) \times[0,1)$ para representar o toro, e $\pi(x, y)=(x(\bmod 1), y(\bmod 1))$, considere o círculo $C=\{(x(\bmod 1), 0) ; x \in \mathbb{R}\} \subset \mathbb{T}^{2} . \alpha x \in \mathbb{Z}$ se, e somente se, $x \in \frac{1}{\alpha} \mathbb{Z}$, assim podemos observar que $\pi(r) \cap C$ é exatamente a órbita de um ponto no círculo $C$ por uma rotação $R_{1 / \alpha}(\theta)=\theta+1 / \alpha(\bmod 1)$ (Figura 2.1$)$, e como $1 / \alpha$ é irracional, essa órbita é densa em $C$, o que nos permite concluir que a imagem da reta $r$ pela aplicação $\pi$ é um subconjunto denso de $\mathbb{T}^{2}$.

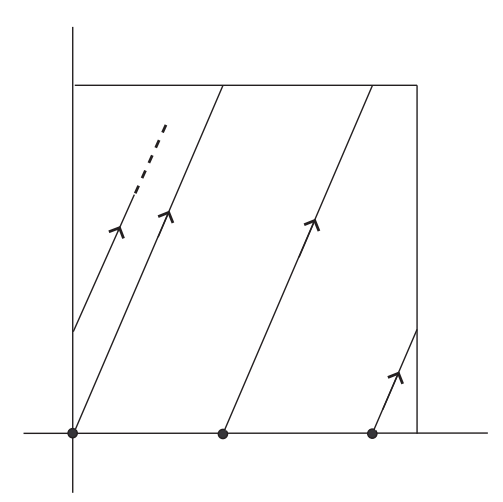

Figura 2.1: Imagem da reta $r$ pela aplicação $\pi$.

Lema 2.3 Seja $g: X \rightarrow \mathbb{R}$ a função de Lyapounov completa descrita no Teorema 2.3. Se definimos $N_{i}=g^{-1}\left(\left(-\infty, c_{i}\right]\right)$, então $f\left(N_{i}\right) \subset \operatorname{int} N_{i}$ e $\Lambda_{i} \subset N_{i}-N_{i-1}$.

Prova: Primeiro vamos verificar que $f\left(N_{i}\right) \subset \operatorname{int} N_{i}$. Se $x \in f\left(N_{i}\right)$, existe um $y \in N_{i}$ com $x=f(y)$. Temos duas possibilidades:

$$
\begin{aligned}
-y \in X \backslash R(f) & \Rightarrow g(x)=g(f(y))<g(y) \in\left(-\infty, c_{i}\right] \\
& \Rightarrow g(x) \in\left(-\infty, c_{i}\right) \\
& \Rightarrow x \in g^{-1}\left(\left(-\infty, c_{i}\right)\right) \subset N_{i} \\
& \Rightarrow x \in \operatorname{int} N_{i} .
\end{aligned}
$$


- $y \in R(f) \Rightarrow x=f(y) \in R(f)$

$$
\Rightarrow g(x)=g(f(y)) \neq c_{i}
$$

utilizando o primeiro item da Observação 2.2,

$$
g(x)=g(f(y)) \leq g(y) \in\left(-\infty, c_{i}\right] \Rightarrow g(x) \in\left(-\infty, c_{i}\right)
$$

ou seja, $x \in \operatorname{int} N_{i}$.

Finalmente provamos que $\Lambda_{i} \subset N_{i}-N_{i-1}$. Temos:

$$
\begin{aligned}
N_{i}-N_{i-1} & =g^{-1}\left(\left(-\infty, c_{i}\right]\right) \cap g^{-1}\left(\left(-\infty, c_{i-1}\right]\right)^{c} \\
& =g^{-1}\left(\left(-\infty, c_{i}\right]\right) \cap g^{-1}\left(\left(c_{i-1},+\infty\right)\right) \\
& =g^{-1}\left(\left(-\infty, c_{i}\right] \cap\left(c_{i-1},+\infty\right)\right) \\
& =g^{-1}\left(\left(c_{i-1}, c_{i}\right]\right) \supset \Lambda_{i} .
\end{aligned}
$$

Quando assumirmos que o conjunto de rotação $\rho(F)$ é um segmento de reta e falarmos que ele tem inclinação irracional, estaremos nos referindo ao coeficiente angular da reta que contém $\rho(F)$.

Proposição 2.3 Sejam $f: \mathbb{T}^{2} \rightarrow \mathbb{T}^{2}$ um homeomorfismo homotópico à identidade, $e$ $F: \mathbb{R}^{2} \rightarrow \mathbb{R}^{2}$ um levantamento. Se $f$ não tem ponto periódico e $\rho(F)$ é um segmento de reta com inclinação irracional, então $\mathbb{T}^{2}$ é transitivo por cadeia por $f$.

Prova: Por contradição suponhamos que $\mathbb{T}^{2}$ não é transitivo por cadeia por $f$. Afirmamos que:

(a) $R(f)$ tem pelo menos duas componentes transitivas por cadeia: Seja $h: \mathbb{T}^{2} \rightarrow \mathbb{R}$ uma função de Lyapounov completa para $f$, e consideremos $r=\sup _{x \in \mathbb{T}^{2}} h(x)$ e $s=i n f_{x \in \mathbb{T}^{2}} h(x)$. É claro que $r \neq s$, caso contrário $\mathbb{T}^{2}$ seria transitivo por cadeia.

Vejamos que $h^{-1}(r)$ e $h^{-1}(s)$ são duas componentes transitivas por cadeia distintas.

- Dado $x \in h^{-1}(r)$ se $x \notin R(f)$, segue que

$$
f^{-1}(x) \notin R(f) \Rightarrow r=h(x)=h\left(f\left(f^{-1}(x)\right)\right)<h\left(f^{-1}(x)\right)
$$

o que contradiz o fato de $r=\sup _{x \in \mathbb{T}^{2}} h(x)$. Logo, se $x \in h^{-1}(r)$, então $x \in R(f)$.

- Seja $x \in h^{-1}(s)$, se $x \notin R(f)$, temos que

$$
f(x) \notin R(f) \Rightarrow s=h(x)>h(f(x))
$$


o que não pode acontecer, já que $s=i n f_{x \in \mathbb{T}^{2}} h(x)$. Deste modo, se $x \in h^{-1}(s)$, então $x \in R(f)$.

Portanto, $h^{-1}(r)$ e $h^{-1}(s)$ são duas componentes transitivas por cadeia, provando (a).

Como $R(f)$ tem mais de uma componente transitiva por cadeia, existe um $\delta>0$ para o qual $R(f)$ tem mais de uma componente $\delta$-transitiva. Sejam $R(f)=\Lambda_{1} \cup \Lambda_{2} \cup \ldots \cup \Lambda_{n}$ a decomposição do conjunto de pontos recorrentes por cadeia em componentes $\delta$-transitivas e $g: \mathbb{T}^{2} \rightarrow \mathbb{R}$ a função de Lyapounov completa como no Teorema 2.3. Escolhemos $g_{0}: \mathbb{T}^{2} \rightarrow \mathbb{R}$ uma aproximação de $g$ de classe $C^{\infty}$ com valores regulares $c_{1}<c_{2}<\ldots<c_{n}$ tal que, a variedade com bordo $M_{i}=g_{0}^{-1}\left(\left(-\infty, c_{i}\right]\right)$ satisfaz

(1) $f\left(M_{i}\right) \subset \operatorname{int} M_{i}$

(2) $\Lambda_{i} \subset M_{i}-M_{i-1}$

Cada bordo $\partial M_{i}$ consiste de uma união disjunta e finita de círculos que são componentes de $g_{0}^{-1}\left(c_{i}\right)$.

(b) Cada componente conexa de $g_{0}^{-1}\left(\left\{c_{1}, c_{2}, \ldots, c_{n}\right\}\right)$ é bordo de um único disco em $\mathbb{T}^{2}$ : De fato, se um desses círculos é essencial, isto é, não é homotópico a um ponto, então $M_{j}$ é um anel essencial em $\mathbb{T}^{2}$ (possivelmente com buracos), para algum $1 \leq j \leq n$ (Figura $2.2)$.

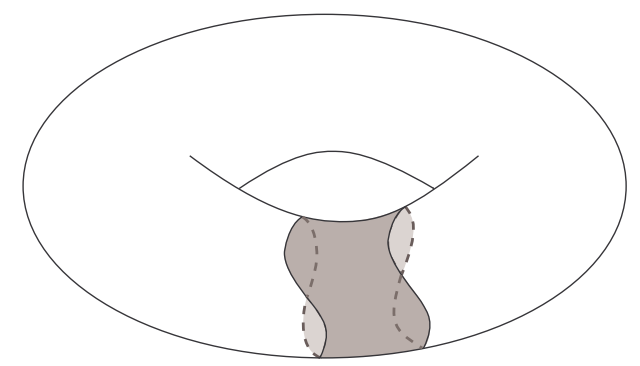

Figura 2.2: Anel essencial no toro $\mathbb{T}^{2}$.

Sejam $\widetilde{M}_{j}$ uma componente conexa de $\pi^{-1}\left(M_{j}\right)$ e $F_{0}$ um levantamento de $f$ de modo que $F_{0}\left(\widetilde{M}_{j}\right) \subset \widetilde{M}_{j}$. Existe $p=\left(p_{1}, p_{2}\right) \in \mathbb{Z}_{+}^{2}$ tal que, dado $\widetilde{x} \in \widetilde{M}_{j}$ existe um único $\widetilde{y} \in \widetilde{M}_{j}$ para o qual $\widetilde{x}=\widetilde{y}+p$. O valor $p_{2} / p_{1}$ nós chamamos de inclinação da faixa $\widetilde{M}_{j}$. Sejam $k, l \subset \mathbb{R}^{2}$ duas retas com inclinação igual a $p_{2} / p_{1}$ tais que $\widetilde{M}_{j}$ esteja contido na região delimitada por estas retas (Figura 2.3).

Seja $v$ um extremo de $\rho\left(F_{0}\right)$, pelo Lema 2.1 , existe $x \in \mathbb{R}^{2}$ tal que

$$
v=\lim _{n \rightarrow+\infty} \frac{F_{0}^{n}(x)-x}{n} .
$$


Podemos supor que $v \neq 0$, caso contrário pelo Lema $2.1 f$ tem ponto fixo. Sendo $v \neq 0$,

$$
\lim _{n \rightarrow+\infty}\left\|F_{0}^{n}(x)-x\right\|=+\infty
$$

Temos duas possibilidades:

(i) Se $x \in \widetilde{M}_{j}$, então $F_{0}^{n}(x) \in \widetilde{M}_{j}$ para cada $n>0$, afirmamos que $v$ é paralelo a $p$, isto é, $v$ tem a mesma inclinação de $\widetilde{M}_{j}$. De fato, se $v$ não é paralelo $p$, então

$$
v \cdot p^{\perp} \neq 0 \text {. }
$$

Logo, existe $n_{0} \in \mathbb{N}$ tal que, para $n \geq n_{0}$,

$$
\left(F_{0}^{n}(x)-x\right) \cdot p^{\perp} \neq 0
$$

isto significa que $F_{0}^{n}(x)-x$ não é paralelo a $p$ para $n \geq n_{0}$. Portanto, como $\left\|F_{0}^{n}(x)-x\right\| \rightarrow$ $+\infty, F_{0}^{n}(x)$ não pertence à faixa delimitada pelas retas $k$ e $l$. Isto contradiz o fato de $F_{0}\left(\widetilde{M}_{j}\right) \subset \widetilde{M}_{j}$ e demonstra que $v$ e $p$ são vetores paralelos (veja a Figura 2.3).

(ii) Se $x \notin \widetilde{M}_{j}$, então $x$ está localizado entre translações de $\widetilde{M}_{j}$ por vetores de $\mathbb{Z}^{2}$, e as iterações $F_{0}^{n}(x)$ se comportam de maneira parecida ao caso em que $x \in \widetilde{M}_{j}$, lembrando que $F_{0}\left(\widetilde{M}_{j}+h\right) \subset \widetilde{M}_{j}+h$ para qualquer $h \in \mathbb{Z}^{2}$.

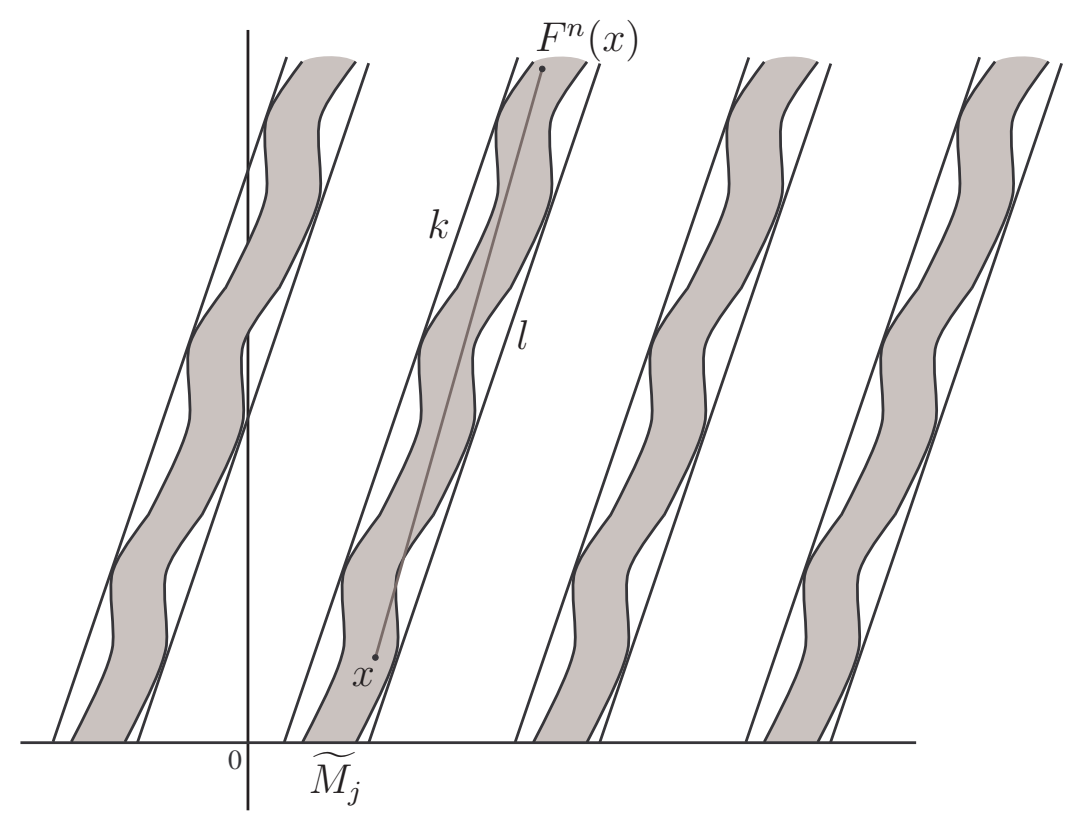

Figura 2.3: $\pi^{-1}\left(M_{j}\right)$ e as retas paralelas com a mesma inclinação de $\widetilde{M}_{j}$.

Se $\omega$ é o outro extremo de $\rho\left(F_{0}\right)$, então, do mesmo modo, temos que $\omega$ é paralelo a $p$. Portanto, como $[v, \omega]=\rho\left(F_{0}\right)$, concluímos que $\rho\left(F_{0}\right)$ tem inclinação $p_{2} / p_{1} \in \mathbb{Q}$, contradizendo a hipótese. Isto prova a afirmação (b). 
De (b), as componentes de bordo de cada $\partial M_{i}$ são não-essenciais, isto é, cada círculo é bordo de um único disco em $\mathbb{T}^{2}$. Como $f\left(M_{i}\right) \subset$ int $M_{i}$ e o número de discos é finito, pelo menos um disco $D$ satisfaz $f^{q}(D) \subset D$ para algum $q \in \mathbb{N}$, e o Teorema do ponto fixo de Brouwer garante que $f^{q}$ tem um ponto fixo, ou seja, $f$ tem um ponto periódico. 


\section{Capítulo 3}

\section{Conjunto de rotação e pontos periódicos}

Este capítulo é dedicado à prova do Teorema Principal desta dissertação, o qual foi obtido por Jonker e Zhang em [12] e estabelece o seguinte:

Teorema Principal Suponha que f é um homeomorfismo do toro $\mathbb{T}^{2}$ homotópico à identidade. Seja $F$ um levantamento de $f$ e suponha que o conjunto de rotação $\rho(F)$ é um segmento de reta com inclinação irracional. Se $\left(p_{1} / n, p_{2} / n\right) \in \rho(F), \operatorname{com} \operatorname{mdc}\left(p_{1}, p_{2}, n\right)=1$, então f tem um ponto periódico de período $n$.

A seguir damos uma breve descrição dos passos fundamentais da prova deste teorema. A prova divide-se em três casos:

Caso 1:O ponto racional $\left(p_{1} / n, p_{2} / n\right)$ é um extremo de $\rho(F)$ : Neste caso a conclusão segue imediatamente do Lema 2.1 (corolário dos Teoremas 2.4 e 3.10 do trabalho de Misiurewicz e Ziemian em [16]).

Caso 2:O ponto racional é a origem e não é um extremo de $\rho(F)$ : Neste caso, primeiro definimos um grafo orientado $G_{n}(\varepsilon)$ (Definição 3.2) onde os vértices orientados são elementos de uma partição do toro $\mathbb{T}^{2}$, e os caminhos orientados representam $\varepsilon$-cadeias. Após estabelecer propriedades de caminhos e laços em $G_{n}(\varepsilon)$ obtemos a Proposição 3.1. Através desta proposição, para qualquer $\varepsilon>0$ nós podemos garantir a existência de uma $\varepsilon$-cadeia periódica da origem. Conseqüentemente, pelo Lema 3.8 (Franks em [7]) a existência do ponto periódico para $f$ é garantida.

Caso 3: O ponto racional não é nulo e não é extremo de $\rho(F)$ : Neste caso, pela Proposição 2.1 nós voltamos ao caso anterior, e a conclusão segue imediatamente. 
No final da Seção 3.2 nós damos um exemplo de um homeomorfismo do toro homotópico à identidade cujo conjunto de rotação é um segmento de reta com inclinação racional que contem infinitos pontos racionais mas que não possui nenhum ponto periódico. Este exemplo é devido a Jonker e Zhang [12].

\subsection{Orafo orientado $G_{n}(\varepsilon)$ de uma $\varepsilon$-partição}

Todos os conceitos e resultados estudados nesta seção são considerando um homeomorfismo $f: \mathbb{T}^{2} \rightarrow \mathbb{T}^{2}$ homotópico à identidade e $F$ um de seus levantamentos.

O resultado fundamental desta seção é a Proposição 3.1. Para enunciar e mostrar esta proposição precisamos definir alguns objetos e estabelecer algumas propriedades.

Primeiro definimos o grafo orientado. Identificamos $\mathbb{T}^{2} \operatorname{com} I^{2}=[0,1) \times[0,1)$. Seja $N$ um inteiro positivo tal que $\varepsilon=1 / N$ seja suficientemente menor que $1 / 8$, e $\|F(x)-F(y)\|<$ $1 / 4$ se $\|x-y\|<\sqrt{2} \varepsilon$, para todo $x, y \in \mathbb{R}^{2}$.

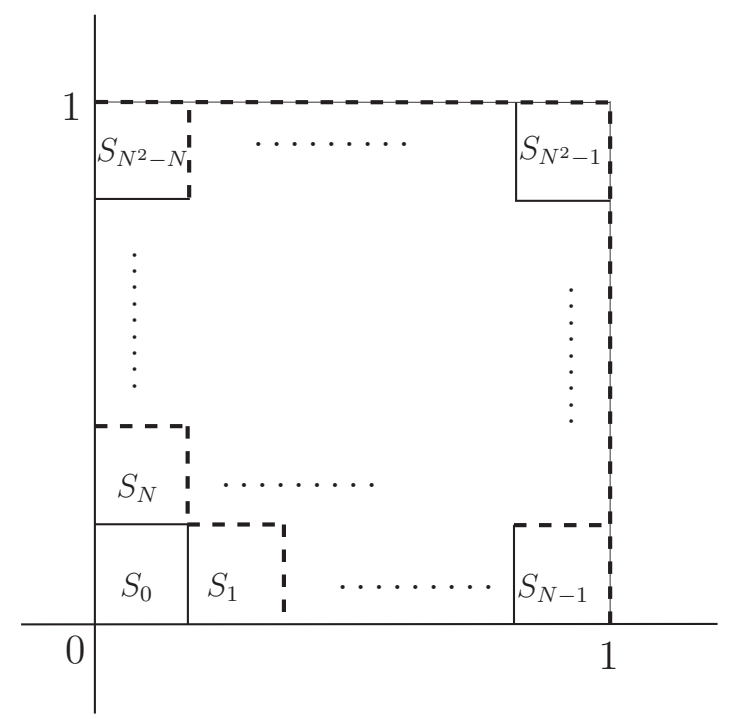

Figura 3.1: $\varepsilon$-partição de $I^{2}$.

Definição 3.1 ( $\varepsilon$-partição de $I^{2}$ ) Particionamos $I^{2}$ em quadrados $S_{i}$ de lado $\varepsilon$, ou seja,

$$
S_{i}=[r / N,(r+1) / N) \times[j / N,(j+1) / N) \text { para } 0 \leq i<N^{2},
$$

$\operatorname{com} i=j N+r$ e $r=i \bmod N$. Os $S_{i}$ são disjuntos e $\bigcup_{i=0}^{N^{2}-1} S_{i}=I^{2}($ Figura 3.1$)$. $\left\{S_{i} ; i=0, \ldots, N^{2}-1\right\}$ é chamada de $\varepsilon$-partição de $I^{2}$. 
Observação 3.1 Note que $\left(S_{i}+h\right) \bigcap\left(S_{i^{*}}+h^{*}\right) \neq \varnothing$ com $0 \leq i, i^{*}<N^{2}$ e $h, h^{*} \in \mathbb{Z}^{2}$ se, e somente se, $S_{i}=S_{i^{*}}$ e $h=h^{*}$.

Definição 3.2 (Grafo de uma $\varepsilon$-partição) Para cada inteiro positivo $n$ tal que $(n+$ $\sqrt{2}) \varepsilon<1 / 8$, nós construímos um grafo orientado $G_{n}(\varepsilon)$, cujos vértices são $\left\{S_{i}\right\}$ e cada aresta orientada é denotada por $e_{i j}$. Onde $e_{i j}=\left(S_{i}, S_{j}\right)$ se, e somente se, existe um ponto $x \in S_{i}$ tal que $d\left(F(x), S_{j}+p_{i j}\right)<n \varepsilon$ para algum $p_{i j} \in \mathbb{Z}^{2}$.

Observação $3.2 O p_{i j} \in \mathbb{Z}^{2}$ associado à aresta $e_{i j}$ na definição anterior é único.

De fato, se $x, y \in S_{i}$ e $p_{i j}, p_{i j}^{*} \in \mathbb{Z}^{2}$ satisfazem $d\left(F(x), S_{j}+p_{i j}\right)<n \varepsilon$ e $d\left(F(y), S_{j}+p_{i j}^{*}\right)<n \varepsilon$, mostraremos que $p_{i j}=p_{i j}^{*}$. Dado $z \in S_{j}$ temos que

$$
\begin{aligned}
d\left(p_{i j}, p_{i j}^{*}\right) & =d\left(p_{i j}+z, p_{i j}^{*}+z\right) \\
& \leq d\left(p_{i j}+z, F(x)\right)+d(F(x), F(y))+d\left(F(y), p_{i j}^{*}+z\right) .
\end{aligned}
$$

Como $p_{i j}+z \in S_{j}+p_{i j}$

$$
\begin{aligned}
d\left(p_{i j}+z, F(x)\right) & \leq d\left(p_{i j}+S_{j}, F(x)\right)+\operatorname{diam}\left(S_{j}+p_{i j}\right) \\
& <(n+\sqrt{2}) \varepsilon .
\end{aligned}
$$

De maneira análoga,

$$
d\left(F(y), p_{i j}^{*}+z\right)<(n+\sqrt{2}) \varepsilon .
$$

Além disto, se $x, y \in S_{j}$ então $d(x, y)<\sqrt{2} \varepsilon$, mais ainda, pela escolha de $\varepsilon$, segue que

$$
d(F(x), F(y))<1 / 4
$$

Portanto,

$$
\begin{aligned}
d\left(p_{i j}, p_{i j}^{*}\right) & <2(n+\sqrt{2}) \varepsilon+1 / 4 \\
& <1 / 2 .
\end{aligned}
$$

Como $p_{i j}, p_{i j}^{*} \in \mathbb{Z}^{2}$, concluímos que $p_{i j}=p_{i j}^{*}$.

Esta observação nos permite definir uma aplicação $\wp$ do conjunto de arestas orientadas de $G_{n}(\varepsilon)$ em $\mathbb{Z}^{2}$ da seguinte maneira:

$$
\wp\left(e_{i j}\right)=p_{i j}
$$




\subsubsection{Caminhos e laços orientados em $G_{n}(\varepsilon)$}

Definição 3.3 (Caminho e laço orientado) Um caminho orientado em $G_{n}(\varepsilon)$ é uma seqüência ordenada finita de arestas orientadas em $G_{n}(\varepsilon)$, tal que o ponto final de cada aresta na seqüência é igual ao ponto inicial da aresta seguinte, exceto na última aresta (Figura 3.2). Um laço em $G_{n}(\varepsilon)$ é um caminho orientado que começa e termina no mesmo vértice. Um laço minimal é um laço em que os únicos vértices comuns são o inicial e o final.

Podemos estender a aplicação $\wp$, definida anteriormente, para o conjunto dos caminhos orientados: Se $\gamma=\left(e_{i_{0} i_{1}}, e_{i_{1} i_{2}}, \ldots, e_{i_{k-1} i_{k}}\right)$ é um caminho orientado em $G_{n}(\varepsilon)$, definimos

$$
\wp(\gamma)=\sum_{j=0}^{k-1} \wp\left(e_{i_{j} i_{j+1}}\right) .
$$
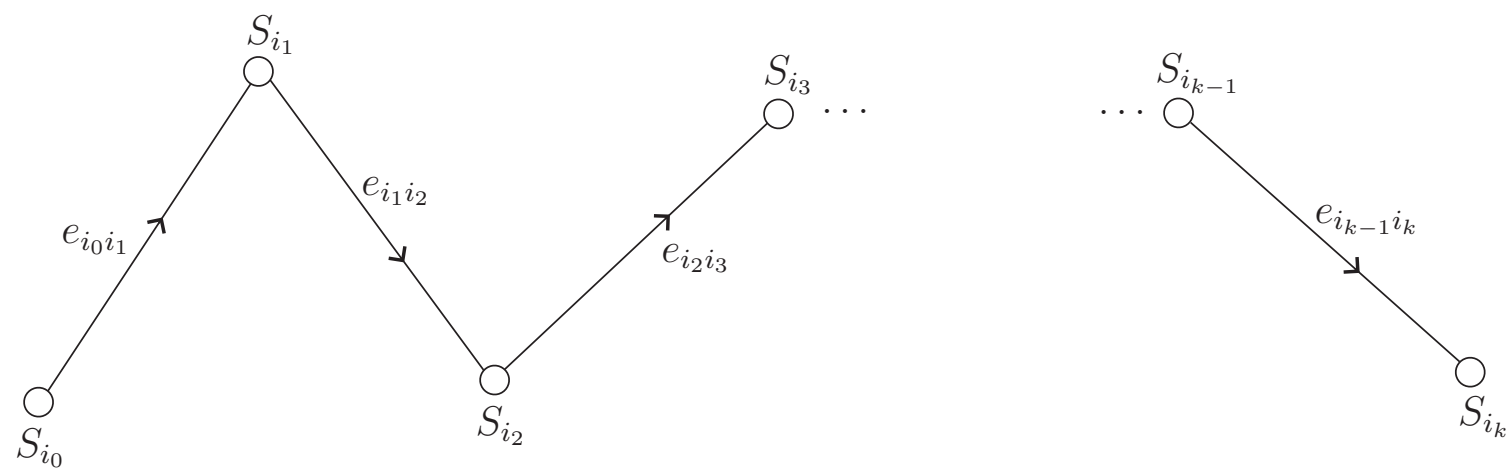

Figura 3.2: Caminho orientado $\gamma=\left(e_{i_{0} i_{1}}, e_{i_{1} i_{2}}, \ldots, e_{i_{k-1} i_{k}}\right)$ em $G_{n}(\varepsilon)$.

Para um ponto $x=\left(x_{1}, x_{2}\right) \in \mathbb{R}^{2}$, denotamos o ponto $\left(x_{1} \bmod \mathbb{Z}, x_{2} \bmod \mathbb{Z}\right)$ por $x \bmod \mathbb{Z}^{2}$. O resultado seguinte estabelece a relação entre caminhos em $G_{n}(\varepsilon)$ e $\delta$-cadeias de $F$.

Lema 3.1 Seja $n \geq 1$ inteiro, $\operatorname{com}(n+\sqrt{2}) \varepsilon<1 / 8$.

(1) Todo caminho orientado $\gamma=\left(e_{i_{0} i_{1}}, e_{i_{1} i_{2}}, \ldots, e_{i_{k-1} i_{k}}\right)$ em $G_{n}(\varepsilon)$ representa uma $\delta$ cadeia $\left\{x_{\alpha}\right\}_{\alpha=0}^{k}$ de $F$ com $\delta=(n+\sqrt{2}) \varepsilon$ e $x_{k}=y_{k}+\wp(\gamma)$, onde $y_{k} \in S_{i_{k}}$ pode ser escolhido arbitrariamente.

(2) Dada uma ne-cadeia de $F$ de $x_{0}$ a $x_{k}$, existe um caminho orientado $\gamma$ em $G_{n}(\varepsilon)$ que corresponde a esta $n \varepsilon$-cadeia e satisfaz $\wp(\gamma)=p_{k}-p_{0}$, onde $p_{k}=x_{k}-\left(x_{k} \bmod \mathbb{Z}^{2}\right)$ 
e $p_{0}=x_{0}-\left(x_{0} \bmod \mathbb{Z}^{2}\right)$. Mais ainda, se $x_{0}=x_{k} \bmod \mathbb{Z}^{2}$, então podemos escolher $\gamma$ sendo um laço.

Prova: (1) Seja $\gamma=\left(e_{i_{0} i_{1}}, e_{i_{1} i_{2}}, \ldots, e_{i_{k-1} i_{k}}\right)$ um caminho orientado em $G_{n}(\varepsilon)$. Para cada $e_{i_{\alpha-1} i_{\alpha}} \operatorname{com} \alpha=1,2, \ldots, k$, existem $y_{\alpha-1} \in S_{i_{\alpha-1}}$ e $p_{i_{\alpha-1} i_{\alpha}} \in \mathbb{Z}^{2}$ tais que

$$
d\left(F\left(y_{\alpha-1}\right), S_{i_{\alpha}}+p_{i_{\alpha-1} i_{\alpha}}\right)<n \varepsilon
$$

conseqüentemente

$$
d\left(F\left(y_{\alpha-1}\right), z_{i_{\alpha}}+p_{i_{\alpha-1} i_{\alpha}}\right)<(n+\sqrt{2}) \varepsilon
$$

para qualquer $z_{i_{\alpha}} \in S_{i_{\alpha}}$.

Definimos $x_{0}=y_{0}$ e $x_{\alpha}=y_{\alpha}+\sum_{j=1}^{\alpha} p_{i_{j-1} i_{j}}$ para $\alpha=1,2, \ldots, k$ com $y_{k} \in S_{i_{k}}$ qualquer. Assim,

$$
\begin{aligned}
d\left(F\left(x_{0}\right), x_{1}\right) & =d\left(F\left(y_{0}\right), y_{1}+p_{i_{0} i_{1}}\right) \\
& <(n+\sqrt{2}) \varepsilon
\end{aligned}
$$

e para $\alpha=1,2, \ldots, k-1$,

$$
\begin{aligned}
d\left(F\left(x_{\alpha}\right), x_{\alpha+1}\right) & =d\left(F\left(y_{\alpha}+\sum_{j=1}^{\alpha} p_{i_{j-1} i_{j}}\right), y_{\alpha+1}+\sum_{j=1}^{\alpha+1} p_{i_{j-1} i_{j}}\right) \\
& =d\left(F\left(y_{\alpha}\right)+\sum_{j=1}^{\alpha} p_{i_{j-1} i_{j}}, y_{\alpha+1}+\sum_{j=1}^{\alpha+1} p_{i_{j-1} i_{j}}\right) \\
& =d\left(F\left(y_{\alpha}\right), y_{\alpha+1}+p_{i_{\alpha} i_{\alpha+1}}\right) \\
& <(n+\sqrt{2}) \varepsilon,
\end{aligned}
$$

ou seja,

$$
d\left(F\left(x_{\alpha}\right), x_{\alpha+1}\right)<(n+\sqrt{2}) \varepsilon
$$

para $\alpha=0, \ldots, k-1$. Portanto $\left\{x_{\alpha}\right\}_{\alpha=0}^{k}$ é uma $\delta$-cadeia de $F \operatorname{com} x_{0}=y_{0}, x_{k}=y_{k}+\wp(\gamma)$ e $\delta=(n+\sqrt{2}) \varepsilon$. Isto prova $(1)$.

(2) Seja $\left\{x_{\alpha}\right\}_{\alpha=0}^{k}$ uma $n \varepsilon$-cadeia de $x_{0}$ a $x_{k}$. Para cada $x_{\alpha}$ existe $p_{\alpha} \in \mathbb{Z}^{2}$ tal que $x_{\alpha} \in$ $S_{i_{\alpha}}+p_{\alpha}$ para algum $S_{i_{\alpha}}$, com isto $x_{\alpha}-p_{\alpha} \in S_{i_{\alpha}}$.

Definimos o caminho orientado $\gamma=\left(e_{i_{0} i_{1}}, e_{i_{1} i_{2}}, \ldots, e_{i_{k-1} i_{k}}\right)$ com a aresta orientada $e_{i_{\alpha} i_{\alpha+1}}$ de $S_{i_{\alpha}}$ a $S_{i_{\alpha+1}} \operatorname{com} \alpha=0, \ldots, k-1$, dada por:

$$
\begin{aligned}
d\left(F\left(x_{\alpha}-p_{\alpha}\right), S_{i_{\alpha+1}}+\left(p_{\alpha+1}-p_{\alpha}\right)\right) & =d\left(F\left(x_{\alpha}\right)-p_{\alpha}, S_{i_{\alpha+1}}+p_{\alpha+1}-p_{\alpha}\right) \\
& =d\left(F\left(x_{\alpha}\right), S_{i_{\alpha+1}}+p_{\alpha+1}\right) \\
& \leq d\left(F\left(x_{\alpha}\right), x_{\alpha+1}\right) \\
& <n \varepsilon
\end{aligned}
$$


para $x_{\alpha}-p_{\alpha} \in S_{i_{\alpha}}$.

Pela definição da aplicação $\wp, \wp\left(e_{i_{\alpha} i_{\alpha+1}}\right)=p_{\alpha+1}-p_{\alpha}$, e

$$
\begin{aligned}
\wp(\gamma) & =\sum_{\alpha=0}^{k-1} \wp\left(e_{i_{\alpha} i_{\alpha+1}}\right) \\
& =\sum_{\alpha=0}^{k-1}\left(p_{\alpha+1}-p_{\alpha}\right) \\
& =p_{k}-p_{0} .
\end{aligned}
$$

Se $x_{0}=x_{k} \bmod \mathbb{Z}^{2}$, então existe $p \in \mathbb{Z}^{2}$ tal que $x_{k}=x_{0}+p$, isto significa que

$$
x_{k} \in\left(S_{i_{0}}+\left(p_{0}+p\right)\right) \bigcap\left(S_{i_{k}}+p_{k}\right),
$$

e pela Observação $3.1, S_{i_{0}}=S_{i_{k}}$ e $p_{k}=p_{0}+p$.. Neste caso, o caminho orientado $\gamma$ é um laço e $\wp(\gamma)=p_{k}-p_{0}=p$.

Dados um caminho orientado $\gamma$ em $G_{n}(\varepsilon)$ e uma $n \varepsilon$-cadeia $\left\{x_{\alpha}\right\}_{\alpha=0}^{k}$ de $F$, fixemos o seguinte:

(1) A $\delta$-cadeia que $\gamma$ representa é a encontrada no Lema 3.1(1);

(2) O caminho orientado que corresponde a $\left\{x_{\alpha}\right\}_{\alpha=0}^{k}$ é aquele que foi encontrado no Lema 3.1(2).

Observação 3.3 Se um caminho orientado $\gamma=\left(e_{i_{0} i_{1}}, e_{i_{1} i_{2}}, \ldots, e_{i_{k-1} i_{k}}\right)$ contém um subcaminho orientado $\gamma_{1}=\left(e_{i_{s} i_{s+1}}, \ldots, e_{i_{r} i_{r+1}}\right)$ que é um laço, isto é, $i_{r+1}=i_{s}$, então as arestas restantes também constituem um caminho $\gamma_{2}=\left(e_{i_{0} i_{1}}, \ldots, e_{i_{s-1} i_{s}}, e_{i_{r+1} i_{r+2}}, \ldots, e_{i_{k-1} i_{k}}\right)$ em $G_{n}(\varepsilon)$. Neste caso, escrevemos $\gamma=\gamma_{1}+\gamma_{2}$.

É fácil notar que, na observação anterior, $\wp(\gamma)=\wp\left(\gamma_{1}\right)+\wp\left(\gamma_{2}\right)$, pois o valor de $\wp$ em um caminho depende apenas das arestas $e_{i_{j} i_{j+1}}$. Conseqüentemente, temos o lema seguinte.

Lema 3.2 Se $\gamma$ é um laço em $G_{n}(\varepsilon)$, então existem laços minimais $\gamma_{1}, \ldots, \gamma_{m}$ tais que

$$
\gamma=\gamma_{1}+\ldots+\gamma_{m}
$$

Observação 3.4 A expressão para $\gamma$ no Lema 3.2 pode não ser única, entretanto o valor de $\sum_{j=1}^{m} \wp\left(\gamma_{j}\right)$ é sempre o mesmo para toda decomposição em laços minimais. 
Definimos os seguintes subconjuntos de $\mathbb{Z}^{2}$ associados aos laços e laços minimais em $G_{n}(\varepsilon)$,

$$
\begin{aligned}
& L_{n}(\varepsilon)=\left\{\wp(\gamma): \gamma \text { é um laço em } G_{n}(\varepsilon)\right\} \\
& M_{n}(\varepsilon)=\left\{\wp(\gamma): \gamma \text { é um laço minimal em } G_{n}(\varepsilon)\right\} \\
& C_{n}(\varepsilon)=\left\{\sum_{i} m_{i} p_{i}: m_{i} \in \mathbb{Z}_{+}, p_{i} \in M_{n}(\varepsilon)\right\}
\end{aligned}
$$

Pelo Lema 3.2, $M_{n}(\varepsilon) \subset L_{n}(\varepsilon) \subset C_{n}(\varepsilon)$, e $M_{n}(\varepsilon)$ é um conjunto finito, pois o número de laços minimais em $G_{n}(\varepsilon)$ é necessariamente finito.

Definição 3.4 Seja $\left\{\gamma_{i}\right\}_{i=1}^{r}$ uma coleção de laços em $G_{n}(\varepsilon)$. Dizemos que $\cup_{i=1}^{r} \gamma_{i}$ é conexa se dados $S_{i}, S_{j}$ dois vértices em $\cup_{i=1}^{r} \gamma_{i}$, existe um caminho com extremos $S_{i}, S_{j}$ contido em $\cup_{i=1}^{r} \gamma_{i}$.

Lema 3.3 Seja $\left\{\gamma_{i}\right\}_{i=1}^{r}$ uma coleção de laços em $G_{n}(\varepsilon)$. Se $\cup_{i=1}^{r} \gamma_{i}$ é conexa, então para todo $m_{i} \in \mathbb{Z}_{+}$, com $m_{i}>0$,

$$
\sum_{i=1}^{r} m_{i} \wp\left(\gamma_{i}\right) \in L_{n}(\varepsilon)
$$

Prova: A prova será feita por indução.

Para $r=1$, basta definir o caminho $\gamma$ que percorre $\gamma_{1}, m_{1}$ vezes, e assim $m_{1} \wp\left(\gamma_{1}\right)=$ $\wp(\gamma) \in L_{n}(\varepsilon)$.

Suponhamos que vale para $r=k$. Dada $\cup_{i=1}^{k+1} \gamma_{i}$ conexa, ao menos um dos subconjuntos $\cup\left\{\gamma_{i}: 1 \leq i \leq k+1, i \neq j\right\}$ para $1 \leq j \leq k+1$ também é conexo. Supomos que $\cup_{i=2}^{k+1} \gamma_{i}$ é conexo e $S_{j}$ é um vértice de $\gamma_{1}$ comum com $\gamma_{2}$.

Pela hipótese de indução $\sum_{i=2}^{k+1} m_{i} \wp\left(\gamma_{i}\right) \in L_{n}(\varepsilon)$, existe um caminho $\gamma$ passando por todas as arestas orientadas de $\cup_{i=2}^{k+1} \gamma_{i}$ um número apropriado de vezes, tal que

$$
\wp(\gamma)=\sum_{i=2}^{k+1} m_{i} \wp\left(\gamma_{i}\right)
$$

Agora construímos um laço $\gamma^{*}$ em $G_{n}(\varepsilon)$ que começa em $S_{j}$ percorre o caminho $\gamma$ retornando a $S_{j}$, e depois percorre o caminho $\gamma_{1}, m_{1}$ vezes, terminando em $S_{j}$. Deste modo

$$
\wp\left(\gamma^{*}\right)=m_{1} \wp\left(\gamma_{1}\right)+\wp(\gamma)=\sum_{i=1}^{k+1} m_{i} \wp\left(\gamma_{i}\right) \in L_{n}(\varepsilon) .
$$




\subsubsection{A componente de 0 em $\mathbb{Z}^{2}$ por $\varepsilon$-cadeias}

Nesta parte do trabalho vamos enunciar e provar a Proposição 3.1. Para isso, analisaremos os pontos de $\mathbb{Z}^{2}$ para os quais a origem pode ser "ligada" por meio de $\varepsilon$-cadeias. Com este intuito, definimos o subconjunto $U(\varepsilon)$ de $\mathbb{Z}^{2}$ :

$$
U(\varepsilon)=\left\{p \in \mathbb{Z}^{2} \text { : existe uma } \varepsilon \text {-cadeia de } F \text { da origem a } p\right\} .
$$

Que chamamos de componente de 0 em $\mathbb{Z}^{2}$ por $\varepsilon$-cadeias. Pelo Lema 3.1, dado $p \in U(n \varepsilon)$ a $n \varepsilon$-cadeia de 0 a $p$ pode ser representada por um laço $\gamma$ em $G_{n}(\varepsilon)$. O seguinte resultado também é conseqüência deste lema.

Corolário 3.1 $U(n \varepsilon) \subset L_{n}(\varepsilon)$ para $n \geq 1$.

Prova: Pela prova do Lema 3.1(2), dado $p \in U(n \varepsilon)$ temos que $x_{k}=p$ e $x_{0}=0, p=0+p$ e com isto $\wp(\gamma)=p$, ou seja, $p \in L_{n}(\varepsilon)$. Portanto $U(n \varepsilon) \subset L_{n}(\varepsilon)$.

Nas provas de alguns lemas seguintes será necessário concatenar $\varepsilon$-cadeias de $F$, por isso é importante vermos a próxima observação.

Observação 3.5 Se existem duas $\varepsilon$-cadeias de $F,\left\{x_{i}\right\}_{i=0}^{k}$ e $\left\{y_{i}\right\}_{i=0}^{r}$ com $x_{k}=y_{0}+p$ para algum $p \in \mathbb{Z}^{2}$, então podemos construir uma $\varepsilon$-cadeia $\left\{z_{i}\right\}_{i=0}^{k+r}$ de $x_{0}$ a $y_{k}+p$ sendo $z_{i}=x_{i}$ para $i=0, \ldots, k$ e $z_{i}=y_{i-k}+p$ para $i=k, \ldots, k+r$.

De fato, para $k \leq i \leq k+r-1$,

$$
\begin{aligned}
d\left(F\left(z_{i}\right), z_{i+1}\right) & =d\left(F\left(y_{i-k}+p\right), y_{i-k+1}+p\right) \\
& =d\left(F\left(y_{i-k}\right)+p, y_{i-k+1}+p\right) \\
& =d\left(F\left(y_{i-k}\right), y_{i-k+1}\right) \\
& <\varepsilon .
\end{aligned}
$$

Lema 3.4 Seja $\mathbb{T}^{2}$ transitivo por cadeia por $f$. Então existe um vetor $\sigma_{1} \in L_{1}(\varepsilon)$ tal que, para toda coleção $p_{j} \in M_{n}(\varepsilon)$ e $m_{j} \in \mathbb{Z}_{+}$, com $n \geq 1$, e $j=1, \ldots, r$,

$$
\begin{gathered}
\sigma_{1}+\sum_{j=1}^{r} m_{j} p_{j} \in U((n+\sqrt{2}) \varepsilon), \text { ou seja } \\
\sigma_{1}+C_{n}(\varepsilon) \subset U((n+\sqrt{2}) \varepsilon) .
\end{gathered}
$$


Prova: $\mathbb{T}^{2}$ transitivo por cadeia implica que: Para cada $0 \leq i<N^{2}$ podemos escolher um $x_{i} \in S_{i}$ tal que existe uma $\varepsilon$-cadeia de $f, \omega_{i}=\left\{x_{0}=0, \ldots, x_{i}, \ldots, x_{k}=0\right\}$. Equivalentemente, existe uma $\varepsilon$-cadeia de $F, \widetilde{\omega}_{i}=\left\{\widetilde{x}_{0}=0, \ldots, \widetilde{x}_{i}, \ldots, \widetilde{x}_{k}=h_{i}\right\}$, para algum $h_{i} \in \mathbb{Z}^{2}$ e com $\widetilde{x}_{i} \in S_{i}+\mathbb{Z}^{2}$. Transladando $\widetilde{\omega}_{1}$ por $h_{0}$, obtemos uma $\varepsilon$-cadeia que liga $h_{0}$ ao ponto $h_{0}+h_{1}$. Pela Observação 3.5 , concatenando esta última com a $\widetilde{\omega}_{0}$, temos uma outra $\varepsilon$-cadeia de 0 a $h_{0}+h_{1}$. Prosseguindo de maneira indutiva, obtemos uma $\varepsilon$-cadeia $\omega$ de 0 a $\sigma_{1}=\sum_{i=0}^{N^{2}-1} h_{i}$. Pelo Lema 3.1(2) existe um laço $\gamma$ em $G_{1}(\varepsilon)$ que corresponde a $\omega$, e $\sigma_{1}=\wp\left(\gamma_{1}^{*}\right) \in U(\varepsilon)$. Observemos que, por construção, $\gamma$ contém todos os vértices $\left\{S_{i}\right\}_{i=0}^{N^{2}-1}$.

Se $p_{j} \in M_{n}(\varepsilon), j=1, \ldots, r$, então existe laço minimal $\gamma_{j}$ em $G_{n}(\varepsilon)$ tal que

$$
\wp\left(\gamma_{j}\right)=p_{j}
$$

Como cada $\gamma_{j}$ tem vértice em comum com $\gamma$, então $\gamma \cup\left(\cup_{j=1}^{r} \gamma_{j}\right)$ é conexa. Logo, pelo Lema 3.3, para $m_{j} \in \mathbb{Z}_{+}$, existe um laço $\gamma^{*}$ em $G_{n}(\varepsilon)$ tal que

$$
\wp\left(\gamma^{*}\right)=\sigma_{1}+\sum_{j=1}^{r} m_{j} p_{j} \in L_{n}(\varepsilon) .
$$

Se $\gamma^{*}=\left(e_{i_{0} i_{1}}, e_{i_{1} i_{2}}, \ldots, e_{i_{k-1} i_{k}}\right)$, podemos supor que $i_{0}=0=i_{k}\left(S_{i_{0}}=S_{i_{k}}=S_{0}\right)$. Deste modo, pelo Lema 3.1(1), temos a $(n+\sqrt{2})$ E-cadeia $\left\{x_{\alpha}\right\}_{\alpha=0}^{k} \operatorname{com} x_{0}=0, x_{k}=y_{k}+\wp\left(\gamma^{*}\right)$ e podemos escolher $y_{k}=0 \in S_{0}$, ou seja, $x_{k}=\wp\left(\gamma^{*}\right)$. Isto mostra que $\wp\left(\gamma^{*}\right) \in U((n+$ $\sqrt{2}) \varepsilon)$.

Lema 3.5 Seja $\mathbb{T}^{2}$ transitivo por cadeia e $\tau$ um dos vetores $\{( \pm 1,0),(0, \pm 1)\}$. Então existem $p \in C_{1}(\varepsilon)$ e uma $2 \varepsilon$-cadeia de 0 a $p+\tau$ cujo laço correspondente $\gamma$ satisfaz $\wp(\gamma)=p+\tau$.

Prova: Vamos provar para $\tau=(1,0)$, sendo que nos outros casos a prova é similar. Considerando $S_{0}, S_{1}, \ldots, S_{N-1}$, definimos $y_{i}=i \varepsilon \tau=(i \varepsilon, 0)$, para $0 \leq i \leq N-1$. Note que $y_{i} \in S_{i}$, pois $S_{i}=[i / N,(i+1) / N) \times[0,1 / N)$ para $0 \leq i \leq N-1($ lembre-se que $\varepsilon=1 / N)$.

Pela transitividade por cadeia, para cada $i=0, \ldots, N-1$ existe $\omega_{i}=\left\{y_{i}, x_{1}^{i}, \ldots, x_{m_{i}}^{i}, y_{i}+\right.$ $\left.p_{i}\right\}, \varepsilon / 2$-cadeia de $F$ de $y_{i}$ a $y_{i}+p_{i}$, para algum $p_{i} \in \mathbb{Z}^{2}$. Pelo Lema 3.1(2) podemos encontrar um laço $\gamma_{i}$ em $G_{1}(\varepsilon)$ que corresponde a $\omega_{i}$ tal que $\wp\left(\gamma_{i}\right)=p_{i}$. 
Observe que, para $i=0, \ldots, N-1$, definindo $y_{N}=(1,0), y_{i+1}=y_{i}+(\varepsilon, 0) \mathrm{e}$ $\widetilde{\omega}_{i}=\left\{y_{i}, x_{1}^{i}, \ldots, x_{m_{i}}^{i}, y_{i+1}+p_{i}\right\}$ é uma $2 \varepsilon$-cadeia de $y_{i}$ a $y_{i+1}+p_{i}$, pois

$$
\begin{aligned}
d\left(F\left(x_{m_{i}}^{i}\right), y_{i+1}+p_{i}\right) & =d\left(F\left(x_{m_{i}}^{i}\right), y_{i}+p_{i}+(\varepsilon, 0)\right) \\
& \leq d\left(F\left(x_{m_{i}}^{i}\right), y_{i}+p_{i}\right)+d\left(y_{i}+p_{i}, y_{i}+p_{i}+(\varepsilon, 0)\right) \\
& <\varepsilon / 2+\varepsilon \\
& <2 \varepsilon .
\end{aligned}
$$

Concatenando as $2 \varepsilon$-cadeias $\left\{\widetilde{\omega}_{i}\right\}_{i=0}^{N-1}$, como na Observação 3.5, encontramos uma $2 \varepsilon$ cadeia $\omega=\left\{y_{0}=0, \ldots, p+(1,0)\right\}$, onde $p=p_{0}+\ldots+p_{N-1}$. Pelo Lema 3.1(2) existe um laço $\gamma$ em $G_{2}(\varepsilon)$ correspondendo a $\omega$ tal que

$$
\wp(\gamma)=\sum_{i=0}^{N-1} p_{i}+(1,0)=p+(1,0) .
$$

Além disto, $p=\sum_{i=0}^{N-1} \wp\left(\gamma_{i}\right) \in C_{1}(\varepsilon)$, pois cada $\gamma_{i}$ é um laço em $G_{1}(\varepsilon)$. Isto conclui a prova.

Corolário 3.2 Seja $\mathbb{T}^{2}$ transitivo por cadeia. Então $C_{2}(\varepsilon)-C_{2}(\varepsilon)=\mathbb{Z}^{2}$.

Prova: Como $\mathbb{Z}^{2}$ pode ser gerado por $\{( \pm 1,0),(0, \pm 1)\}$ sobre $\mathbb{Z}_{+}$, então será suficiente mostrar que $m \tau_{1}+k \tau_{2} \in C_{2}(\varepsilon)-C_{2}(\varepsilon)$, para quaisquer $m, k \in \mathbb{Z}_{+}$e $\tau_{1}, \tau_{2} \in\{( \pm 1,0),(0, \pm 1)\}$. De fato, pelo Lema 3.5, dados $\tau_{1}, \tau_{2} \in\{( \pm 1,0),(0, \pm 1)\}$ existem $p_{1}, p_{2} \in C_{1}(\varepsilon)$ e laços $\gamma_{1}$, $\gamma_{2}$ em $G_{2}(\varepsilon)$ com

$$
\wp\left(\gamma_{1}\right)=p_{1}+\tau_{1} \text { e } \wp\left(\gamma_{2}\right)=p_{2}+\tau_{2} .
$$

Como $\wp\left(\gamma_{1}\right), \wp\left(\gamma_{2}\right) \in L_{2}(\varepsilon) \subset C_{2}(\varepsilon)$, pela definição de $C_{2}(\varepsilon)$ e $C_{1}(\varepsilon)$, para quaisquer $m, k \in \mathbb{Z}_{+}$,

$$
m\left(p_{1}+\tau_{1}\right)+k\left(p_{2}+\tau_{2}\right) \in C_{2}(\varepsilon) \text { e } m p_{1}+k p_{2} \in C_{1}(\varepsilon)
$$

isto implica que

$$
m\left(p_{1}+\tau_{1}\right)+k\left(p_{2}+\tau_{2}\right)-\left(m p_{1}+k p_{2}\right)=m \tau_{1}+k \tau_{2} \in C_{2}(\varepsilon)-C_{1}(\varepsilon)
$$

e como $C_{1}(\varepsilon) \subset C_{2}(\varepsilon)$ segue que $m \tau_{1}+k \tau_{2} \in C_{2}(\varepsilon)-C_{2}(\varepsilon)$. Portanto,

$$
C_{2}(\varepsilon)-C_{2}(\varepsilon)=\mathbb{Z}^{2}
$$

No lema seguinte, · denota o produto interno usual de $\mathbb{R}^{2}$. 
Lema 3.6 Suponhamos que $[0, v] \subseteq \rho(F)$ tem inclinação irracional e que v é um extremo de $\rho(F)$. Se $\xi$ é um funcional linear não-trivial de $\mathbb{R}^{2}$ em $\mathbb{R}$ com $\xi(v)=0$, então existem $p, q \in C_{1}(\varepsilon)$ tais que $\xi(p)>0, \xi(q)<0$ e $p \cdot v>0, q \cdot v<0$.

Prova: Seja $\widetilde{C}_{n}(\varepsilon)$ o conjunto gerado por $M_{n}(\varepsilon)$ sobre $\mathbb{R}_{+}$. Então $\widetilde{C}_{n}(\varepsilon)$ é um cone convexo e fechado (possivelmente todo o plano).

Primeiramente vamos mostrar que $v \in \widetilde{C}_{1}(\varepsilon)$. De fato, como $v$ é um ponto extremo de $\rho(F)$, segue do Lema 2.1 que existe um ponto $x \in \mathbb{T}^{2}$ tal que $v=\rho(F, x)$, ou seja, tomando $\widetilde{x} \in \pi^{-1}(x)$,

$$
v=\lim _{m \rightarrow \infty} \frac{F^{m}(\widetilde{x})-\widetilde{x}}{m} .
$$

Para cada $m \geq 0, F^{m}(\widetilde{x}) \in S_{m}^{*}+p_{m}$, onde $\left\{S_{m}^{*}\right\} \subset\left\{S_{i}\right\}$ e $p_{m} \in \mathbb{Z}^{2}$.

$$
F^{m}(\widetilde{x})-\widetilde{x} \in\left(S_{m}^{*}+p_{m}\right)-\left(S_{0}^{*}+p_{0}\right)
$$

isto é,

$$
F^{m}(\widetilde{x})-\widetilde{x}=\left(s_{m}^{*}+p_{m}\right)-\left(s_{0}^{*}+p_{0}\right),
$$

com $s_{m}^{*} \in S_{m}^{*}$ e $s_{0}^{*} \in S_{0}^{*}$. Fazendo $s_{m}^{*}-s_{0}^{*}=t_{m}$ temos que

$$
F^{m}(\widetilde{x})-\widetilde{x}=p_{m}-p_{0}+t_{m}, \operatorname{com}\left\|t_{m}\right\|<\sqrt{2} .
$$

Utilizando a $\varepsilon$-cadeia $\left\{F^{i}(\widetilde{x})\right\}_{i=0}^{m}$, pelo Lema 3.1(2) existe um caminho orientado $\gamma_{m}$ em $G_{1}(\varepsilon)$ que corresponde a esta $\varepsilon$-cadeia e $\wp\left(\gamma_{m}\right)=p_{m}-p_{0}$. Se nós "removemos" todos os laços contidos em $\gamma_{m}$, como na Observação 3.3, os vértices restantes constituem um caminho orientado $\gamma^{*}$ em $G_{1}(\varepsilon)$. Deste modo,

$$
\frac{F^{m}(\widetilde{x})-\widetilde{x}}{m}=\frac{1}{m} L_{m}+\frac{1}{m} \wp\left(\gamma^{*}\right)+\frac{1}{m} t_{m}
$$

onde $L_{m} \in C_{1}(\varepsilon) \subset \widetilde{C}_{1}(\varepsilon)$. Como $\gamma^{*}$ consiste de vértices distintos, $\wp\left(\gamma^{*}\right)$ é limitado e o valor que o limita não depende de $m$. Fazendo $m \rightarrow \infty$, segue que

$$
\frac{1}{m} \wp\left(\gamma^{*}\right) \rightarrow 0 \text { e } \frac{1}{m} t_{m} \rightarrow 0, \text { pois }\left\|t_{m}\right\|<\sqrt{2},
$$

conseqüentemente $\frac{1}{m} L_{m} \rightarrow v$. Sabemos que $\frac{1}{m} L_{m} \in \widetilde{C}_{1}(\varepsilon), \widetilde{C}_{1}(\varepsilon)$ é fechado e $\frac{1}{m} L_{m} \rightarrow v$, portanto $v \in \widetilde{C}_{1}(\varepsilon)$.

Afirmamos que $v \in \operatorname{int} \widetilde{C}_{1}(\varepsilon)$. De fato, se $\widetilde{C}_{1}(\varepsilon) \neq \mathbb{R}^{2}$ e $v \notin i n t \widetilde{C}_{1}(\varepsilon)$ então $v$ está na fronteira de $\widetilde{C}_{1}(\varepsilon)$, ou seja, $v$ pertence a uma reta que passa pela origem e por um ponto 
$h \in M_{1}(\varepsilon) \subset \mathbb{Z}^{2}$. Seja $h=(a, b)$ com $a, b \in \mathbb{Z}, a \neq 0$, deste modo o segmento de reta entre a origem e $v$ teria inclinação racional (Figura 3.3), o que contradiz a hipótese e mostra que $v \in \operatorname{int} \widetilde{C}_{1}(\varepsilon)$.

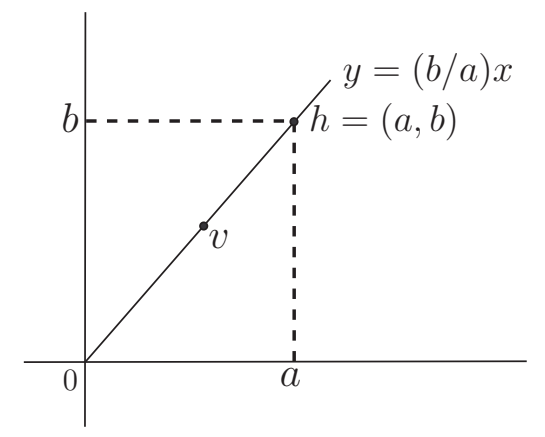

Figura 3.3: Segmento com inclinação racional.

Como $v \in \operatorname{int} \widetilde{C}_{1}(\varepsilon)$, existe uma bola aberta de centro em $v$ contida em $\widetilde{C}_{1}(\varepsilon)$. Com isto, sabendo que $\xi$ é não-trivial e $\xi(v)=0$, podemos escolher $p, q \in \widetilde{C}_{1}(\varepsilon)$ satisfazendo

$$
\xi(p)>0, \xi(q)<0, p \cdot q>0, p \cdot v>0, q \cdot v<0
$$

onde $p=\sum_{i} r_{i} p_{i}$ e $q=\sum_{i} r_{i}^{*} q_{i} \operatorname{com} p_{i}, q_{i} \in M_{1}(\varepsilon)$ e $r_{i}, r_{i}^{*} \in \mathbb{Q}_{+}$.

Finalmente afirmamos que $p, q \in C_{1}(\varepsilon)$. Multiplicando por um inteiro positivo suficientemente grande para eliminar os denominadores de todos os $r_{i}$ 's, $r_{i}^{*}$ 's, obtemos os vetores que renomeamos como $p, q$ pertencentes a $C_{1}(\varepsilon)$ satisfazendo (3.1). Isto conclui a prova.

Dado $y \in \mathbb{R}^{2}$ denotamos por $\measuredangle(y, v)$ o ângulo entre $y$ e $v$, com isto, para $0 \leq \theta<\pi / 2$ e $r>0$, definimos o conjunto $E(v, \theta, r)$ associado com o vetor rotação $v$ do Lema 3.6 como (veja a Figura 3.4)

$$
E(v, \theta, r)=\left\{y \in \mathbb{R}^{2}:\|y\| \geq r \text { e } \measuredangle(y, v)<\theta\right\}
$$

Se $p, q$ são os dois pontos encontrados no Lema 3.6, então eles são linearmente independentes, e como $M_{2}(\varepsilon)$ é finito, temos a seguinte observação.

Observação 3.6 (1) Existe $H=\left\{h_{1}, h_{2}, \ldots, h_{m}\right\} \subset \mathbb{Z}^{2}$ um conjunto de representantes das classes laterais de $\mathbb{Z}^{2}$ em $p \mathbb{Z}+q \mathbb{Z}$ tal que para cada $i=1, \ldots, m, h_{i}=m_{i} p+n_{i} q$, para alguns $m_{i}, n_{i} \in \mathbb{Z}_{+}$. 
(2) Seja $M_{2}(\varepsilon)=\left\{p_{1}, p_{2}, \ldots, p_{s}\right\}$. Se $\mathbb{T}^{2}$ é transitivo por cadeia, então pelo Corolário 3.2 podemos escrever $h_{i}=\sum_{j=1}^{s} \lambda_{i j} p_{j}$, para $\lambda_{i j} \in \mathbb{Z}$ e $p_{j} \in M_{2}(\varepsilon) \subset C_{2}(\varepsilon)$. Considere $\lambda_{j}=\max _{1 \leq i \leq m}\left|\lambda_{i j}\right|$, e $\sigma_{2}=\sum_{j=1}^{s} \lambda_{j} p_{j}$, isto será utilizado na demonstração do próximo lema.

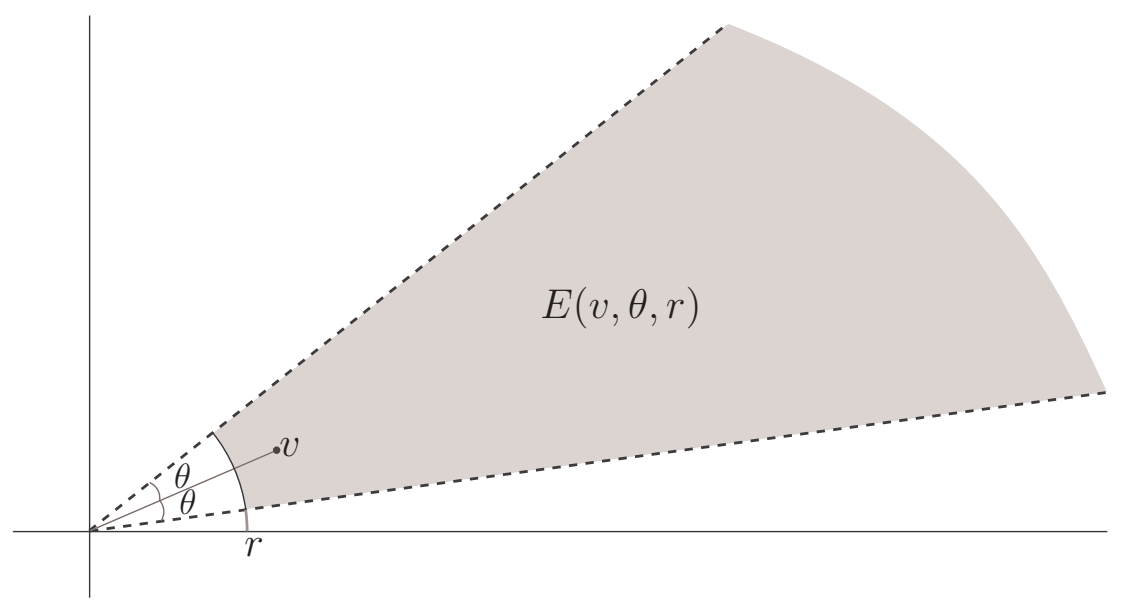

Figura 3.4: O conjunto $E(v, \theta, r)$.

Lema 3.7 Se $\mathbb{T}^{2}$ é transitivo por cadeia, então existem $\theta>0$ e $r>0$ tais que

$$
E(v, \theta, r) \bigcap \mathbb{Z}^{2} \subset C_{2}(\varepsilon) .
$$

Prova: Seja $K=\sigma_{2}+p \mathbb{R}_{+}+q \mathbb{R}_{+}$, com $\sigma_{2}$ sendo o vetor que foi definido anteriormente. Podemos encontrar um $\theta>0$ tão pequeno quanto o necessário e um $r$ grande tais que $E(v, \theta, r) \subset K$. Para concluirmos a demonstração basta mostrar que $K \cap \mathbb{Z}^{2} \subset C_{2}(\varepsilon)$. Se $u \in K \bigcap \mathbb{Z}^{2}$, então $u=\sigma_{2}+h_{i}+r_{1} p+r_{2} q$ para algum $h_{i} \in H$ e $r_{1}, r_{2} \in \mathbb{Z}_{+}$. Pela maneira como $\sigma_{2}$ foi construído, $\lambda_{j}+\lambda_{i j} \geq 0$ para $1 \leq j \leq s$. Isto implica que $\sigma_{2}+h_{i}=\sum_{j=1}^{s}\left(\lambda_{j}+\lambda_{i j}\right) p_{j} \in C_{2}(\varepsilon)$, e como $p, q \in C_{1}(\varepsilon) \subset C_{2}(\varepsilon)$, concluímos que $u \in C_{2}(\varepsilon)$.

Proposição 3.1 Se $\mathbb{T}^{2}$ é transitivo por cadeia, então existem $\theta>0$ e $r>0$ tais que

$$
E(v, \theta, r) \bigcap \mathbb{Z}^{2} \subset U(4 \varepsilon) .
$$

Prova: Sejam $\theta_{0}$ e $r_{0}$ os valores encontrados no Lema 3.7. Evidentemente, podemos encontrar $\theta<\theta_{0}$ e $r>r_{0}$ de maneira que $E(v, \theta, r) \subset \sigma_{1}+E\left(v, \theta_{0}, r_{0}\right)$, sendo $\sigma_{1}$ o vetor definido no Lema 3.4. Então pelos Lemas 3.7 e 3.4,

$$
E(v, \theta, r) \bigcap \mathbb{Z}^{2} \subset \sigma_{1}+E\left(v, \theta_{0}, r_{0}\right) \bigcap \mathbb{Z}^{2} \subset \sigma_{1}+C_{2}(\varepsilon) \subset U((2+\sqrt{2}) \varepsilon) \subset U(4 \varepsilon) .
$$




\subsection{Prova do Teorema Principal}

O lema seguinte será utilizado na demonstração do Teorema Principal, e pode ser encontrado no trabalho de Franks em [7].

Lema 3.8 Seja F um levantamento de um homeomorfismo do toro $\mathbb{T}^{2}$ homotópico à identidade. Se para todo $\varepsilon>0$ existe uma $\varepsilon$-cadeia periódica para $F$, então $F$ tem um ponto fixo.

Agora, com os resultados que obtivemos até aqui já podemos fazer a prova do Teorema Principal.

Teorema Principal Suponha que f é um homeomorfismo do toro $\mathbb{T}^{2}$ homotópico à identidade. Seja F um levantamento de $f$ e suponha que o conjunto de rotação $\rho(F)$ é um segmento de reta com inclinação irracional. Se $\left(p_{1} / n, p_{2} / n\right) \in \rho(F), \operatorname{com} \operatorname{mdc}\left(p_{1}, p_{2}, n\right)=1$, então f tem um ponto periódico de período $n$.

Prova: Denotamos por $[v, \omega]$ o segmento $\rho(F)$, onde $v$ e $\omega$ são os dois pontos extremos do comjunto de rotação, e seja $u=\left(p_{1} / n, p_{2} / n\right) \in \rho(F)$ o ponto racional. Dividiremos a prova em três casos.

CASO 1: Se $u=v$ ou $u=\omega$, então $u$ é um ponto extremo do conjunto de rotação $\rho(F)$ e o resultado segue do Lema 2.1.

Nos casos em que $u$ não é um extremo de $\rho(F)$ a prova será feita por contradição, assumindo que $f$ não tem ponto periódico.

CASO 2: $u=0$ não é um extremo de $\rho(F)$. Pela Proposição 2.3, como $f$ não tem ponto periódico, $\mathbb{T}^{2}$ é transitivo por cadeia. Dado $\varepsilon>0$ qualquer, tomamos um $\delta=1 / N<\varepsilon / 4$ para um inteiro positivo $N$ suficientemente grande. Pela Proposição 3.1, existem $\theta_{i}, r_{i}>$ $0, i=1,2$, tais que

$$
\begin{gathered}
E\left(v, \theta_{1}, r_{1}\right) \cap \mathbb{Z}^{2} \subset U(4 \delta) \subset U(\varepsilon) \\
\mathrm{e} \\
E\left(\omega, \theta_{2}, r_{2}\right) \cap \mathbb{Z}^{2} \subset U(4 \delta) \subset U(\varepsilon) .
\end{gathered}
$$

$\operatorname{Sejam} \theta=\min \left\{\theta_{1}, \theta_{2}\right\}$ e $r=\max \left\{r_{1}, r_{2}\right\}$, deste modo $E(v, \theta, r) \subset E\left(v, \theta_{1}, r_{1}\right)$ e $E(\omega, \theta, r) \subset$ $E\left(\omega, \theta_{2}, r_{2}\right)$. Conseqüentemente, 


$$
[E(v, \theta, r) \cup E(\omega, \theta, r)] \cap \mathbb{Z}^{2} \subset U(\varepsilon)
$$

Além disto, como $0 \in[v, \omega]$, dado $p \in E(v, \theta, r) \cap \mathbb{Z}^{2}$ segue que $-p \in E(\omega, \theta, r) \cap \mathbb{Z}^{2}$ (veja a Figura 3.5), existem $\varepsilon$-cadeias $\alpha_{1}$ de 0 a $p$ e $\alpha_{2}$ de 0 a $-p$. Mais ainda, transladando $\alpha_{2}$ por $p$ obtemos uma $\varepsilon$-cadeia $\alpha_{3}$ de $p$ a 0 , pois $F(x+p)=F(x)+p$ para qualquer $x \in \mathbb{R}^{2}$. Concatenando $\alpha_{1}$ e $\alpha_{3}$ (ver Observação 3.5) obtemos uma $\varepsilon$-cadeia periódica de 0. Logo, pelo Lema 3.8, $F$ tem um ponto fixo. Isto contradiz a nossa suposição.

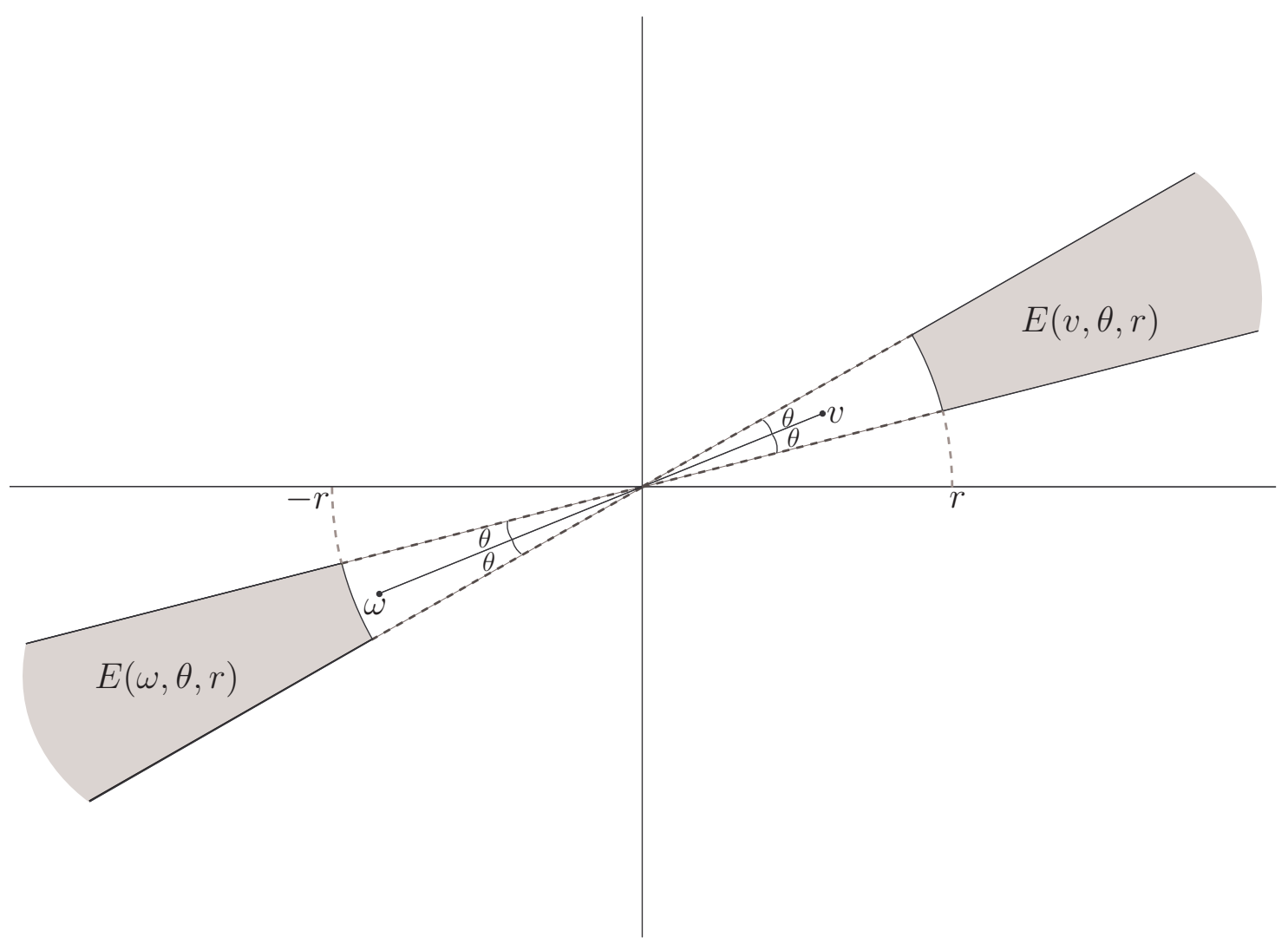

Figura 3.5: O conjunto $E(\omega, \theta, r) \cup E(v, \theta, r)$.

CASO 3: $u \neq 0$ não é um extremo de $\rho(F)$. Escrevemos $u=\frac{1}{n} p$ onde $p=\left(p_{1}, p_{2}\right) \in \mathbb{Z}^{2}$ e $\operatorname{mdc}\left(p_{1}, p_{2}, n\right)=1$. Como $f$ não tem ponto periódico, $f^{n}$ também não tem. É claro que $G=F^{n}-p$ é um levantamento de $f^{n}$, e pela Proposição $2.1, \rho(G)=n \rho(F)-p$, ou seja, $\rho(G)$ é um segmento de reta que tem a mesma inclinação de $\rho(F)$. Além disto, $0=n u-p \in \rho(G)$. Deste modo, utilizando o argumento do caso 2 para $f^{n}$ e seu levantamento $G$, concluímos que $G$ tem um ponto fixo. E novamente chegamos a uma contradição. 
Finalmente, sabendo que $f$ tem um ponto periódico $x$, vamos mostrar que o período é $n$. Seja $k \in \mathbb{Z}_{+}$tal que $f^{k}(x)=x$, e escolhemos um $\widetilde{x} \in \mathbb{R}^{2}$ satisfazendo $\pi(\widetilde{x})=x$. Afirmamos que $\rho(F, x)=u$. De fato, como $f^{k}(\pi(\widetilde{x}))=\pi(\widetilde{x})$ e $f^{k} \circ \pi=\pi \circ F^{k}$, logo $\pi(\widetilde{x})=\pi\left(F^{k}(\widetilde{x})\right)$, isto é,

$$
F^{k}(\widetilde{x})=\widetilde{x}+h
$$

para algum $h \in \mathbb{Z}^{2}$. Conseqüentemente,

$$
F^{r k}(\widetilde{x})=\widetilde{x}+r h, \forall r \in \mathbb{Z}_{+}
$$

Dado $0 \leq i<k$, lembrando que qualquer inteiro positivo pode ser escrito como $r k+i$ para algum $r \in \mathbb{Z}_{+}$,

$$
F^{r k+i}(\widetilde{x})=F^{i}\left(F^{r k}(\widetilde{x})\right)=F^{i}(\widetilde{x}+r h)=F^{i}(\widetilde{x})+r h
$$

e deste modo,

$$
\frac{F^{r k+i}(\widetilde{x})-\widetilde{x}}{r k+i}=\frac{F^{i}(\widetilde{x})+r h-\widetilde{x}}{r k+i}=\frac{F^{i}(\widetilde{x})-\widetilde{x}}{r k+i}+\frac{r h}{r k+i}
$$

Portanto,

$$
\rho(F, x)=\lim _{s \rightarrow \infty} \frac{F^{s}(\widetilde{x})-\widetilde{x}}{s}=\frac{1}{k} h \in \rho(F) \bigcap \mathbb{Q}^{2} .
$$

Como o segmento de reta $\rho(F)$ tem inclinação irracional, segue que $\rho(F, x)=u$. Conseqüentemente, como $h=\frac{k}{n} p \in \mathbb{Z}^{2}$, pelo Lema 1.1, existe $m \in \mathbb{Z}_{+}$tal que $k=m n$.

Já vimos que $F^{k}(\widetilde{x})-h=\widetilde{x}$, com isto

$$
F^{m n}(\widetilde{x})-m p=\widetilde{x}
$$

Dado $y \in \mathbb{R}^{2}$,

$$
\left(F^{n}-p\right)^{r}(y)=F^{r n}(y)-r p, \forall r \in \mathbb{Z}_{+} .
$$

Temos que $\left(F^{n}-p\right)^{m}(\widetilde{x})=F^{m n}(\widetilde{x})-m p=\widetilde{x}$, isto é, $F^{n}-p$ tem um ponto periódico, e pelo Teorema 1.2 (Brouwer) $F^{n}-p$ tem um ponto fixo, portanto $f^{n}$ possui um ponto fixo, ou seja, $f$ tem um ponto periódico de período $n$. Isto conclui a prova o Teorema Principal.

A seguir veremos um exemplo de um homeomorfismos $f$ homotópico à identidade com levantamento $F$ tal que o conjunto de rotação $\rho(F)$ é um segmento de reta com inclinação 
racional, contém infinitos pontos racionais e não possui nenhum ponto periódico.

Exemplo: Seja $X$ um campo de vetores $C^{\infty}$ em $\mathbb{T}^{2}$ com as seguintes propriedades:

(a) X não tem singularidades;

(b) $X$ tem exatamente duas órbitas periódicas $\alpha$ e $\beta$, as quais têm períodos irracionais. Além disto, $\alpha$ é atratora e $\beta$ repulsora;

(c) Sejam $A=\{(x, 1 / 2): x \in \mathbb{R}\}$ e $B=\{(x, 0): x \in \mathbb{R}\}$. Existe $\widetilde{X}$ um campo de vetores em $\mathbb{R}^{2}$ que é um levantamento de $X$ tal que $\pi(A)=\alpha, \pi(B)=\beta,\left.\widetilde{X}\right|_{A}=a$ e $\left.\tilde{X}\right|_{B}=b$.

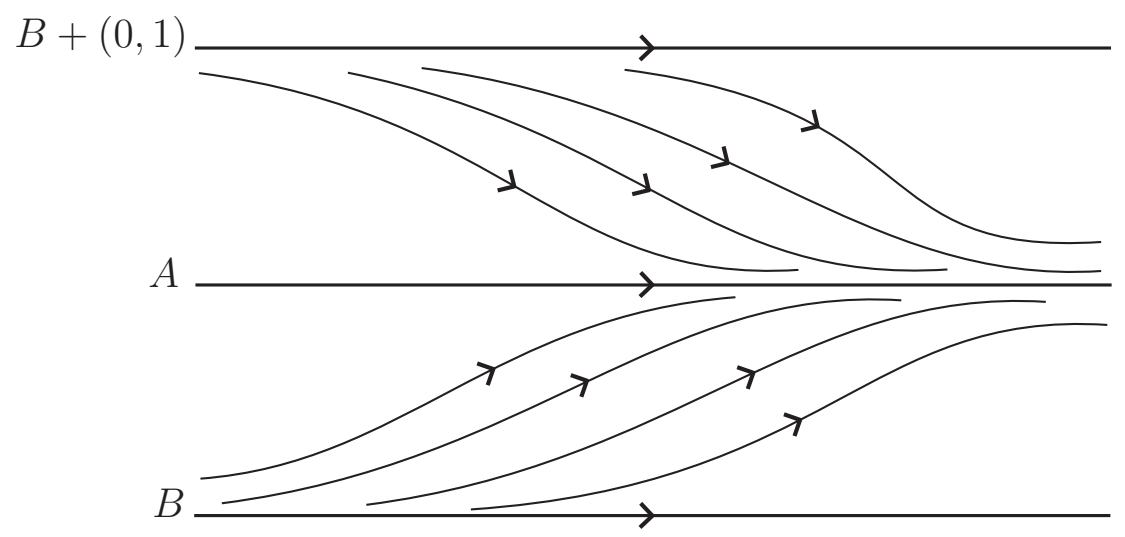

Figura 3.6: Campo de vetores $\tilde{X}$.

Se $\widetilde{X}^{t}$ é o fluxo de $\widetilde{X}$, então, pelo item $(\mathrm{c}), \widetilde{X}^{t}(p)=p+t a$ e $\widetilde{X}^{t}(q)=q+$ ta para cada $t \in \mathbb{R}, p \in A$ e $q \in B$. Em particular, se $F=\widetilde{X}^{1}$ (tempo 1 do fluxo $\widetilde{X}^{t}$ ), então

$$
F(p)=p+a \text { para cada } p \in A \text { e } F(q)=q+b \text { para cada } q \in B .
$$

Conseqüentemente,

$$
\frac{F^{n}(p)-p}{n}=\frac{p+n a-p}{n}=a \quad \text { e } \quad \frac{F^{n}(q)-q}{n}=\frac{q+n b-q}{n}=b .
$$

pois $F(A) \subset A$ e $F(B) \subset B$. Isto mostra que $a, b \in \rho(F)$, portanto, como o conjunto de rotação é convexo, $[a, b] \subset \rho(F)$.

Vamos verificar que $\rho(F)=[a, b]$. Por contradição, suponhamos que $\rho(F) \nsubseteq[a, b]$, isto significa que existe $\omega \in \mathbb{R}^{2} \backslash[a, b]$ que é um extremo de $\rho(F)$. Conseqüentemente, pelo 
Lema 2.1, existe $x_{0} \in \mathbb{R}^{2}$ tal que

$$
\omega=\lim _{n \rightarrow+\infty} \frac{F^{n}\left(x_{0}\right)-x_{0}}{n},
$$

como $\frac{1}{n} x_{0} \rightarrow 0$ quando $n \rightarrow+\infty$, temos que

$$
\omega=\lim _{n \rightarrow+\infty} \frac{F^{n}\left(x_{0}\right)}{n}
$$

Por outro lado, seja $\varepsilon_{0}>0$ tal que $\|\omega-a\|>\varepsilon_{0}$. Então para cada $p \in A$ fixo, existe $n_{0} \in \mathbb{N}$ tal que

$$
n>n_{0} \Rightarrow\left\|F^{n}\left(x_{0}\right)-F^{n}(p)\right\| \geq n \varepsilon_{0} .
$$

Como $F$ é um levantamento de um homeomorfismo do toro, $F^{n}$ é uniformemente contínua, ou seja, dado $\varepsilon \in\left(0, \varepsilon_{0}\right)$ existe $\delta=\delta(n)>0$ tal que, para quaisquer $x, y \in \mathbb{R}^{2}$

$$
\|x-y\|<\delta \Rightarrow\left\|F^{n}(x)-F^{n}(y)\right\|<\varepsilon
$$

Transladando $x_{0}$, se necessário, podemos assumir que $x_{0}$ esteja na faixa definida entre as retas $B$ e $B+(0,1)$. Seja $n>n_{0}$, como $A$ é atrator, podemos supor que $\left\|x_{0}-p\right\|<\delta$, conseqüentemente, de (3.3) segue que

$$
\left\|F^{n}\left(x_{0}\right)-F^{n}(p)\right\|<\varepsilon
$$

Isto contradiz (3.2), mostrando que $\rho(F)=[a, b] \subset \mathbb{R} \times\{0\}$.

Em conclusão, por construção $f=X^{1}$ (tempo um do fluxo $X^{t}$ de $X$ ) não tem pontos periódicos, porém, seu levantamento $F$ é tal que $\rho(F)$ é um segmento de reta com inclinação racional e contém infinitos pontos racionais. 


\section{Referências Bibliográficas}

[1] BAI-LIN, H. Elementary Symbolic Dynamics and chaos in dissipative systems. Singapura: World Scientific, 1989.

[2] BERGE, M. M.; WALKER, R. B. Periodic point free maps of tori which have rotation sets with interior. Nonlinearity, v. 6, n. 3, pp. 481-489, 1993.

[3] BROWN, M. A new proof of Brouwer's lemma on translation arcs. Houston Journal of Mathematics, v. 10, n. 1, pp. 35-41, 1984.

[4] CONLEY, C. Isolated Invariant Sets and the Morse Index. CBMS Regional Conf. in Math., 38. American Mathematical Society, Providence, R. I., 1978.

[5] DEVAnEY, R. L. An Introduction to Chaotic Dynamical Systems. 2.ed. EddisonWesley, 1995.

[6] FATHI, A. An orbit closing proof of Brouwer's lemma on translation arcs. L'Enseignement Mathématique, v. 33, n. 3-4, pp. 315-322, 1987.

[7] FRANKS, J. Realizing rotation vectors for torus homeomorphisms. Trans. Amer. Math. Soc. 311 (1989), pp. 107-116.

[8] FRANKS, J. A variation on the Poincaré-Birkhoff Theorem. Hamiltonian dynamical systems. Conteporary Mathematics, v. 81, Amer. Math. Soc., Providence, R. I., pp. 111-117, 1988.

[9] FRANKS, J. The rotation sets and periodics points for torus homeomorphisms. Dynamical Systems and Chaos, World Scientific, River Edge, NJ, pp. 41-48, 1995.

[10] HANDEL, M. Periodic point free homeomorphism of $\mathbb{T}^{2}$. Porc. Amer. Math. Soc., v. 107, n. 2, pp. 511-515, 1989. 
[11] HANDEL, M. The rotation set of a homeomorphism of the annulus is closed. Comm. Math. Phys., v. 127, n. 2, pp. 339-349, 1990.

[12] JONKER, L. B.; ZHANG, L. Torus homeomorphisms whose rotation sets have empty interior. Ergodic Theory \& Dynamical Systems, v. 18, n. 5, pp. 1173-1185, 1998.

[13] KATOK, A.; HASSELBLATT, B. Introduction to the Modern Theory of Dynamical Systems. Cambrige University press, 1995.

[14] LIMA, ELON L. Curso de análise. 8 ed. Rio de Janeiro: Impa, v. 2, 2005.

[15] LLIBRE, J.; MACKAY, R.S. Rotation vectors and entropy for homeomorphisms of the torus isotopic to the identity. Ergodic Theory \& Dynamical Systems, v. 11, n.1, pp. 115-128, 1991.

[16] MISIUREWICZ, M.; ZIEMIAN, K. Rotation sets for maps of tori. J. London Math. Soc. 40 (1989), pp. 490-506.

[17] MISIUREWICZ, M.; ZIEMIAN, K. Rotation sets and ergodic measures for torus homeomorphisms. Fundamenta Mathematicae, v. 137, n. 1, pp. 45-52, 1991.

[18] POINCARÉ, H. Ouevres complètes, tome 1. Gauthier-Villars, Paris, pp. 137-158, 1952 .

[19] WILSON, R. J. Introduction to graph theory. Edimburgo: Oliver \& Boyd, 1972. 


\section{Índice Remissivo}

$\varepsilon$-cadeia, 18

de Poincaré, 12

$\varepsilon$-partição, 28

do ponto fixo de Brouwer, 5

Caminho orientado, 30

Principal, 40

Componente

Vetor rotação, 16

$\delta$-transitiva, 21

transitiva por cadeia, 20

Conjunto

de rotação, 15

transitivo por cadeia, 19

Função de Lyapounov completa, 20

Grafo, 6

de uma $\varepsilon$-partição, 29

orientado, 6

Grau, 8

Laço, 30

minimal, 30

Levantamento, 7, 15

Número

de rotação, 12

de translação, 12

Recorrente por cadeia, 18

Teorema

de Brouwer, 6

de Conley, 21 